Pacific Journal of Mathematics

A PARTIAL DIFFERENTIAL EQUATION ARISING IN 


\title{
A PARTIAL DIFFERENTIAL EQUATION ARISING IN CONFORMAL MAPPING
}

\author{
P. R. GARABEDIAN
}

1. Introduction. During the past few years considerable attention has been given to the role played by kernel functions in conformal mapping, potential theory, and the theory of linear partial differential equations of elliptic type. The interest in this study has originated from the unifying influence which the concept of a kernel function introduces in these theories, and from the simple relationships discovered between the various kernels and the classical Green's and Neumann's functions $[3-5,8,13]$. Wuch of the older theory has been given a new interpretation, and a new light has been shed upon the study of canonical conformal maps, the Dirichlet problem, and the fundamental existence theorems $[9,10,12]$. The methods and results, which received their original impetus from investigations of functions of several complex variables [1], have been surprisingly simple and basic.

The present paper is devoted to the study of several kernel functions which arise in conformal mapping and in mathematical physics, and to the investigation of some eigenvalue problems related to these kernels. We show that the kernel function associated with the norm

$$
\iint_{D}|\phi(z)|^{2} \rho(z) d x d y
$$

of analytic functions $\phi(z)$ of the complex variable $z$ in a plane domain $D$ can be expressed in terms of the Green's function of the partial differential equation

$$
\frac{\partial}{\partial \bar{z}} \frac{1}{\rho(z)} \frac{\partial}{\partial z} S(z)=0
$$

where

$$
\frac{\partial}{\partial z}=\frac{1}{2}\left(\frac{\partial}{\partial x}-i \frac{\partial}{\partial y}\right), \quad \frac{\partial}{\partial \bar{z}}=\frac{1}{2}\left(\frac{\partial}{\partial x}+i \frac{\partial}{\partial y}\right), \quad z=x+i y .
$$


Ilere $\rho(z)$ is a positive weight function. Such weighted norms arise in the theory of orthogonal polynomials and the study of problems in interpolation and approximation $[15,16]$. A similar discussion of the kernel for the norm

$$
\iint_{D} u(z)^{2} \rho(z) d x d y
$$

of real harmonic functions $u(z)$ in $D$ is carried out.

These kernels are found to be related, in the case $\rho \equiv 1$, to the eigenfunctions of the extremal problem

$$
\frac{\left|\iint_{D} w(z)^{2} d x d y\right|}{\iint_{D}|w(z)|^{2} d x d y}=\text { maximum, } w(z) \text { analytic in } D,
$$

introduced by Friedrichs [7]. This eigenvalue problem is shown to be equivalent to two other eigenvalue problems of a quite different character.

Underlying the manipulations is the idea that minimum problems formultiple integrals with differential equations as side conditions, such as those mentioned above, correspond always to boundary value problems of a very simple nature in the theory of partial differential equations. Proofs are carried out in such a way as to yield the existence of the solutions of the related boundary value problems. ${ }^{1}$

An application of the theoretical material is made to the case where $D$ is an ellipse. In particular, it is found that the Green's function of a convex clamped plate need not be of one sign.

2. The partial differential equation. Let $\rho(z)$ be a positive continuously differentiable function of $x$ and $y$ in a region containing the plane domain $D$ of finite connectivity and its boundary $C$. We shall suppose that $C$ consists of $n$ simple closed curves $C_{1}, \cdots, C_{n}$ which have continuous curvature. We define $\Omega$ to be the class of functions $\phi(z)$ which are analytic in $D$ and have a finite norm

$$
\iint_{D}|\phi(z)|^{2} \rho(z) d \sigma, \quad d \sigma=d x d y .
$$

It is not hard to show [1] that there exists in $\Omega$ a complete orthonormal system $\left\{\phi_{\nu}(z)\right\}$,

${ }^{1}$ For quite similar existence proofs, cf. the works $[19,20,21]$ of G. Fichera, at the end of the table of references, to which the author's attention has recently been drawn. 


$$
\iint_{D} \phi_{\nu}(z) \overline{\phi_{\mu}(z)} \rho(z) d \sigma=\delta_{\nu \mu}= \begin{cases}0, & (\nu \neq \mu), \\ 1, & (\nu=\mu),\end{cases}
$$

and that every function $\phi(z) \in \Omega$ has an expansion

$$
\phi(z)=\sum_{\nu=1}^{\infty} a_{\nu} \phi_{\nu}(z), \quad a_{\nu}=\iint_{D} \phi(z) \overline{\phi_{\nu}(z)} \rho(z) d \sigma,
$$

which converges uniformly in each closed subdomain of $D$.

We define the kernel function $K(z, t)$ of the class $\Omega$ by the formula

$$
K(z, t)=\sum_{\nu=1}^{\infty} \phi_{\nu}(z) \overline{\phi_{\nu}(t)} .
$$

Clearly, $K(z, t)$ is analytic in $z$ and $\bar{t}$, and

$$
\phi(z)=\iint_{D} K(z, t) \phi(t) \rho(t) d \sigma,
$$

The reproducing property (3) characterizes $K(z, t)$ completely, and therefore the kernel function is independent of the orthonormal system $\left\{\phi_{\nu}(z)\right\}$. From (3) and the Schwarz inequality

$$
\begin{aligned}
|\phi(z)|^{2}=\left|\iint_{D} K \phi \rho d \sigma\right|^{2} & \leq \iint_{D}|K(z, t)|^{2} \rho d \sigma \iint_{D}|\phi|^{2} \rho d \sigma \\
& =K(z, z) \iint_{D}|\phi(t)|^{2} \rho(t) d \sigma,
\end{aligned}
$$

we deduce an important extremal property of the function

$$
f(z)=\frac{K(z, t)}{K(t, t)} \in \Omega .
$$

Indeed, $f(z)$ yields the minimum of the norm (1) amongst all functions $\phi \in \Omega$ with $\phi(t)=1$. The minimum value of the norm is $K(t, t)^{-1}$.

Our main objective is to determine the analytic function $K(z, t)$ from considerations which are independent of orthonormal systems or reproducing and minimum 
properties, and are based rather upon a Dirichlet boundary value problem. We shall proceed in such a manner that we obtain simultaneously the desired formula for the kernel function and the existence of a solution of the pertinent boundary value problem.

We suppose that $w$ is a point in the exterior $B$ of $D$. We set

$$
\phi(z)=\frac{1}{z-w}
$$

in the integral equation (3), and we obtain

$$
I(w, t)=\iint_{D} \frac{K(t, z) \rho(z) d \sigma}{z-w}=\frac{1}{t-w}, \quad w \in B .
$$

Now for $w \in D$, we find, by Poisson's equation,

$$
\begin{aligned}
\frac{\partial I(w, t)}{\partial \bar{w}} & =2 \frac{\partial^{2}}{\partial \bar{w} \partial w} \iint_{D} K(t, z) \rho(z) \log \frac{1}{|z-w|} d \sigma \\
& =-\pi \rho(w) K(t, w) .
\end{aligned}
$$

But since $K(t, w)$ is analytic in $\bar{w}$, this yields for $I(w, t)$ the complex second order partial differential equation

$$
\frac{\partial}{\partial w} \frac{1}{\rho(w)} \frac{\partial}{\partial \bar{w}} I(w, t)=0, \quad w \in D
$$

Since $I(w, t)$ satisfies this partial differential equation, or, rather, system of two partial differential equations, of elliptic type in $D$, and since the explicit formula (4) holds in $B$, we are led to attempt to prove that $I(w, t)$ is continuous across $C$ and therefore solves the boundary value problem suggested by (4).

We proceed to prove the continuity of $I(w, t)$ at a point $w_{0} \in C$. Since $C$ has continuous curvature, there is a circle $\Gamma$ lying in $D$ and tangent to $C$ at $w_{0}$. We denote by $w$ a point of $\Gamma$ on the normal to $C$ through $w_{0}$, and we denote by $w^{*}$ the inverse of $w$ in the circle $\Gamma$. We first show that

$$
\begin{aligned}
I_{1}\left(w, w^{*}\right) & =\iint_{\Gamma} K(t, z)\left\{\frac{1}{z-w}-\frac{1}{z-w^{*}}\right\} d \sigma \\
& =\iint_{\Gamma} K(t, z) \frac{d}{d z} \log \frac{z-w}{z-w^{*}} d \sigma=0 .
\end{aligned}
$$


Indeed, the linear fractional transformation

$$
\zeta=\frac{z-w}{z-w^{*}}
$$

carries $\Gamma$ into a circle $\gamma$ centered at the origin in the $\zeta-p l a n e$, and in terms of the new variable $\zeta$, the function $I_{1}\left(w, w^{*}\right)$ has the form

$$
\begin{aligned}
I_{1}\left(w, w^{*}\right) & =\iint_{\gamma} K\left(t, z(\zeta) \overline{z^{\prime}(\zeta)} \frac{d}{d \zeta} \log \zeta d \sigma(\zeta)\right. \\
& =\iint_{\gamma} K(t, z(\zeta)) \overline{z^{\prime}(\zeta)} \frac{d \sigma(\zeta)}{\zeta} .
\end{aligned}
$$

By expanding $K(t, z(\zeta)) \overline{z^{\prime}(\zeta)}$ in a Taylor's series of powers of $\bar{\zeta}$ in $\gamma$ and using polar coordinates and the orthogonality of the sine and cosine functions, we find readily that the latter integral vanishes.

We have, by (6),

$$
\begin{aligned}
I(w, t)-I\left(w^{*}, t\right) & =\iint_{D} \frac{K(t, z)\left(w-w^{*}\right) \rho(z) d \sigma}{(z-w)\left(z-w^{*}\right)} \\
& =\iint_{D-\Gamma} \frac{K(t, z)\left(w-w^{*}\right) \rho(z) d \sigma}{(z-w)\left(z-w^{*}\right)} \\
& \quad+\iint_{\Gamma} \frac{K(t, z)\left(w-w^{*}\right)(\rho(z)-\rho(w)) d \sigma}{(z-w)\left(z-w^{*}\right)}=I^{\prime}+I^{\prime \prime}
\end{aligned}
$$

The integrand of $I^{\prime}$ is dominated in $D-\Gamma$ by an expression

$$
\frac{A|K(t, z)|}{\left|z-w_{0}\right|}
$$

since $\left|z-w_{0}\right| /|z-w|$ and $\left|w-w^{*}\right| /\left|z-w^{*}\right|$ are bounded there. This estimate of the integrand of $I^{\prime}$ is integrable over $D-\Gamma$ by the Schwarz inequality

$$
\left(\iint_{D-\Gamma} \frac{|K|}{\left|z-w_{0}\right|} d \sigma\right)^{2} \leq \iint_{D-\Gamma}|K|^{2} d \sigma \iiint_{D-\Gamma} \frac{d \sigma}{\left|z-w_{0}\right|^{2}},
$$

where the last integral converges because of the deletion of the circle $\Gamma$ from $D$. Furthermore, the integrand in $I^{\prime \prime}$ is dominated by an integrable expression $B|K(t, z)|$, 
since $\rho(z)$ satisfies a Lipschitz condition

$$
|\rho(z)-\rho(w)| \leq B^{\prime}|z-w| \text {. }
$$

Thus we can apply the Lebesgue convergence theorem to the integrals $l^{\prime}$ and $l^{\prime \prime}$ to obtain

$$
\lim _{w \rightarrow w_{0}}\left[I(w, t)-I\left(w^{*}, t\right)\right]=\iint_{D} \lim _{w \rightarrow w_{0}}\left\{\frac{K(t, z)\left(w-w^{*}\right)}{(z-w)\left(z-w^{*}\right)}\right\} \rho(z) d \sigma=0 .
$$

Since we see from (4) that $I(w, t)$ is uniformly continuous for $w \in B$, it is possible to conclude from the special continuity condition (7), in which $w$ and $w^{*}$ are inverse points in $\Gamma$, that $l(w, t)$ is, in fact, continuous across $C$ with no restriction.

Fith these calculations, at hand, we set

$$
S(w, t)=\iint_{D} \frac{K(t, z) \rho(z) d \sigma}{z-w}+\frac{1}{w-t}, \quad w \in D,
$$

and from (4) and the continuity of $I(w, t)$ we find

$$
\lim _{w \rightarrow C} S(w, t)=0
$$

while by (5)

$$
\frac{\partial}{\partial w} \frac{1}{\rho(w)} \frac{\partial}{\partial \bar{w}} S(w, t)=0, \quad w \in D
$$

Hence $S(w, t)$ is the solution of the elliptic partial differential equation (5) in $D$ which has zero boundary values and has a simple pole at $w=t \in D$. We have now a new characterization of the kernel function $K(w, t)$ in terms of the equation (5), for Poisson's equation yields the representation

$$
\overline{K(w, t)}=-\frac{1}{\pi \rho(w)} \frac{\partial S(w, t)}{\partial \bar{w}} .
$$

Note that since $K(w, t)$ is given in terms of a first derivative of $S(w, t)$, the second order partial differential equation (5) for $S$ becomes the Cauchy-Riemann differential equations for the analytic function $K(w, t)$.

Wie denote by $G(w, t)$ the Green's function in $D$ of the equation

$$
\frac{\partial}{\partial \bar{w}} \frac{1}{\rho(w)} \frac{\partial}{\partial w} u(w)=0
$$


adjoint to (5). That is, $G(w, t)$ satisfies (9) in $D$ except at the point $w=t$, where it has a singularity

$$
G(w, t)=\rho(t) \log \frac{1}{|w-t|}+\text { continuous terms, }
$$

and $G(w, t)$ vanishes for $w \in C$. Since $\rho(w)$ is real, the conjugate $\overline{u(w)}$ of a solution $u(w)$ of (9) is a solution of the adjoint equation (5), and conversely. Hence we obtain, comparing singularities and boundary values,

$$
\overline{S(w, t)}=\frac{2}{\rho(t)} \frac{\partial G(w, t)}{\partial \bar{t}},
$$

and therefore, finally,

$$
K(w, t)=-\frac{2}{\pi \rho(w) \rho(t)} \frac{\partial^{2} G(w, t)}{\partial w \partial \bar{t}} .
$$

It is natural to consider, in addition to $K(w, t)$, various other analytic functions in $D$ defined by minimum problems for the norm (1) and closely connected with the kernel function as given by (10). Let $\beta$ be a simple closed curve in $D$ and let $\Omega_{\beta}$ consist of all functions $\phi \in \Omega$ possessing in $D$ indefinite integrals which are single-valued except for the one period

$$
\oint_{\beta^{\prime}} \phi(z) d z=1
$$

taken over all paths $\beta^{\prime}$ homologous to $\beta$ in $D$. We denote by $W_{\beta}(z)$ the function in $\Omega_{\beta}$ which minimizes (1). Hence

$$
\iint_{D}^{n} \overline{W_{\beta}(z)} \phi(z) \rho(z) d \sigma=0
$$

for every function $\phi \in \Omega$ with a single-valued indefinite integral in $D$. We define the generalized harmonic measure $\omega_{\beta}(z)$ by the formula

$$
\omega_{\beta}(w)=\iint_{D} \frac{W_{\beta}(z) \rho(z) d \sigma}{\bar{z}-\bar{w}},
$$

and we find by application of Poisson's equation and continuity considerations, as in the preceding, that $\omega_{\beta}(w)$ satisfies the elliptic equation (9) and has constant boundary values on each component of $C$. 
It becomes clear, then, that our procedure is sufficient for a complete discussion of minimum problems in the norm (1), and we turn to the study of the system of elliptic equations (9).

3. The energy integral. Let $u(z)$ and $v(z)$ be any pair of suitably differentiable functions in $D+C$. Then Green's theorem yields the basic identities

$$
\begin{aligned}
& \iint_{D} u \frac{\partial}{\partial z} \frac{1}{\rho} \frac{\partial v}{\partial \bar{z}} d \sigma+\iint_{D} \frac{1}{\rho} \frac{\partial u}{\partial z} \frac{\partial v}{\partial \bar{z}} d \sigma=-\frac{1}{2 i} \oint_{C} \frac{u}{\rho} \frac{\partial v}{\partial \bar{z}} d \bar{z}, \\
& \iint_{D} \frac{\partial}{\partial \bar{z}} \frac{1}{\rho} \frac{\partial u}{\partial z} d \sigma+\iint_{D} \frac{1}{\rho} \frac{\partial u}{\partial z} \frac{\partial v}{\partial \bar{z}} d \sigma=\frac{1}{2 i} \oint_{C} \frac{v}{\rho} \frac{\partial u}{\partial z} d z .
\end{aligned}
$$

Thus it is clear that (9) is the Euler equation of the integral

$$
\iint \frac{1}{\rho(z)}\left|\frac{\partial u(z)}{\partial z}\right|^{2} d \sigma=\frac{1}{4} \iint \frac{1}{\rho}\left\{\left(a_{x}+b_{y}\right)^{2}+\left(a_{y}-b_{x}\right)^{2}\right\} d \sigma, u=a+i b,
$$

and (5) and (9) are adjoint partial differential equations. If $u(z)$ is a solution of (9), and $v(z)$ is a solution of (5), in a region bounded by curves $\alpha$, we obtain the formula

$$
\oint_{\alpha}\left\{\frac{u}{\rho} \frac{\partial v}{\partial \bar{z}} d \bar{z}+\frac{v}{\rho} \frac{\partial u}{\partial z} d z\right\}=0 .
$$

Setting $u(z)=G(z, t)$ and $v(z)=\overline{G(z, w)}$ and applying (13), we have the symmetry rule

$$
G(w, t)=\overline{G(t, w)} .
$$

Thus the Green's function $G(w, t)$ of (9) is, as a function of $t$, the Green's function of (5). Finally, we have the Green's formula

$$
u(w)=\frac{1}{\pi i} \oint_{C} \frac{u(z)}{\rho(z)} \frac{\partial G(w, z)}{\partial \bar{z}} d \bar{z}
$$

for the solution of the Dirichlet boundary value problem for (9). Since $G(w, z)=0$, $z \in C$, this formula can be written

$$
u(w)=\frac{1}{2 \pi} \oint_{C} \frac{u(z)}{\rho(z)} \frac{\partial G^{\prime}(w, z)}{\partial \nu} d s
$$


where $s$ represents the arc length and $\nu$ the inner normal of $C$, with a similar formula for the solution of (5).

The general solution $u(w)$ of the elliptic partial differential equation (9) can be obtained directly using the evident connection with the Cauchy-Riemann differential equations. We find

$$
u(w)=\iint_{D} \frac{f(z) \rho(z) d \sigma}{\bar{z}-\bar{w}}+\overline{g(w)}
$$

where $f(z)$ and $g(w)$ are arbitrary analytic functions. Note that

$$
\frac{1}{\rho(w)} \frac{\partial u(w)}{\bar{\partial} w}=-\pi f(w)
$$

and note that the expression

$$
\frac{1}{2 \pi} \iint \frac{\rho(z) d \sigma}{(z-t)(\bar{z}-\bar{w})}
$$

is a basic fundamental solution of (9). Each solution of (5) is the complex conjugate of a solution of (9), and thus we have, for the general solution $v(w)$ of (5),

$$
v(w)=\iint_{D} \frac{\overline{h(z)} \rho(z) d \sigma}{z-w}+j(w),
$$

where $h(z)$ and $j(w)$ are analytic. Therefore we find for the scalar product between $u(w)$ and $v(w)$ in terms of the energy integral (12) the representation

$$
\iint_{D} \frac{1}{\rho} \frac{\partial u}{\partial w} \frac{\partial v}{\partial \bar{w}} d \sigma=\pi^{2} \iint_{D} f(w) \overline{h(w)} \rho d \sigma
$$

as the scalar product of the functions $f, h \in \Omega$. Thus the norm (1) and the energy integral (12) are equivalent,

$$
\iint_{D} \frac{1}{\rho}\left|\frac{\partial u(z)}{\partial z}\right|^{2} d \sigma=\pi^{2} \iint_{D} \rho(z)|f(z)|^{2} d \sigma .
$$

The classical approach to the equation (9) through the Dirichlet principle would consist in seeking a function $u(w)$ minimizing (12) in a class of differentiable functions with prescribed boundary values. Our attack is based rather upon extremal problems for ( 1 ) in the class $\Omega$, and contrasts with the older method in that we 
operate, in a sense, entirely within the family of solutions of (9). Note that the formulation in terms of (1) imposes on $\rho(z)$ only the conditions of positiveness and continuity, except in the neighborhood of $C$, where a Lipschitz or Ilölder condition is necessary for the continuity proof (7).

Let us study a special case of the theory developed so far. Let $\mu(z)$ be a function analytic in $D+C$, and set

$$
\rho(z)=|\mu(z)|^{2} .
$$

The general solution of (9) can now be written

$$
u(w)=\overline{\mu(w)} U(w),
$$

where $U(w)$ is complex and harmonic. Indeed,

$$
\frac{\partial}{\partial \bar{w}} \frac{1}{|\mu(w)|^{2}} \frac{\partial}{\partial w} \overline{\mu(w)} U(w)=\frac{\partial}{\partial \bar{w}} \frac{1}{\mu(w)} \frac{\partial U(w)}{\partial w}=\frac{1}{\mu(w)} \frac{\partial^{2} U(w)}{\partial \bar{w} \partial w}=0 .
$$

Note in passing that for $\mu(z)=z$ the system of two second order equations (9) can be reduced to the one fourth order elasticity equation, which has a general solution $u_{1}(z)$ of the form

$$
u_{1}(z)=R\{\bar{z} U(z)\}
$$

If $g(w, t)$ denotes the Green's function of the Laplace equation in $D$, we obtain

$$
G(w, t)=\overline{\mu(w)} \mu(t) g(w, t) .
$$

Thus by (10) the kernel function $K(w, t)$ is given by

$$
\begin{aligned}
K(w, t) & =-\frac{2}{\pi|\mu(w) \mu(t)|^{2}} \frac{\partial^{2}}{\partial w \partial \bar{t}}[\overline{\mu(w)} \mu(t) g(w, t)] \\
& =-\frac{2}{\pi \mu(w) \overline{\mu(t)}} \frac{\partial^{2} g(w, t)}{\partial w \bar{t}}=\frac{k(w, t)}{\mu(w) \overline{\mu(t)}},
\end{aligned}
$$

where $k(w, t)$ is the kernel function of (1) with $\rho \equiv 1$. This result has a simple meaning when $\mu(w)$ is the derivative of a conformal mapping $\zeta=M(w)$ of the domain $D$,

$$
\frac{d \zeta}{d w}=M^{\prime}(w)=\mu(w)
$$


Here (17) merely expresses the conformal invariance of the kernel function $k(w, t)$ as a differential

$$
K(w, t) d M(w) \overline{d M(t)}=k(w, t) d w \overline{d t},
$$

since the weight $\rho(w)=|\mu(w)|^{2}$ is the Jacobian of the mapping $\zeta=M(w)$.

It is of interest to remark at this point that the differential equation (9) exhibits a certain invariance under conformal mappings $\zeta=M(w)$. Take, again, an arbitrary weight function $\rho(w)$ and assume that it behaves as an absolute invariant under the transformation $\zeta=M(w)$. In the $\zeta$-plane we have

$$
\frac{\partial}{\partial \zeta}=\frac{\partial}{M^{\prime}(w) \partial w}, \frac{\partial}{\partial \bar{\zeta}}=\frac{\partial}{\overline{M^{\prime}(w)} \partial \bar{w}}, \quad \zeta=M(w)
$$

and therefore

$$
\frac{\partial}{\partial \bar{\zeta}} \frac{1}{\rho} \frac{\partial}{\partial \zeta}=\frac{1}{M^{\prime}(w)} \frac{\partial}{\partial \bar{w}} \frac{1}{\rho} \frac{1}{M^{\prime}(w)} \frac{\partial}{\partial w}=\frac{1}{\left|M^{\prime}(w)\right|^{2}} \frac{\partial}{\partial \bar{w}} \frac{1}{\rho} \frac{\partial}{\partial w} .
$$

Since this operator changes only by the multiplicative factor $\left|M^{\prime}(w)\right|^{-2}$ when we transform from the $w$-plane to the $\zeta$-plane, the invariance of the class of solutions of (9) under conformal mapping follows. Clearly, the kernel function $K(w, t)$ with scalar weight $\rho$ varies as a differential in both arguments.

In closing this section, we call attention to the fact that the Neumann's function of (9) plays no role in the study of the kernel function with a general weight function $\rho(z)$, although when $\rho(z)$ is constant on $C$ the problem of determining solutions of (9) in $D$ with prescribed normal derivatives on $C$ has significance.

4. Expansion of Green's function. We turn our attention to the expansion of the Green's function of (9) in terms of an orthonormal system $\left\{\phi_{\nu}(z)\right\}$ complete in $\Omega$. We obtain a construction of $G(w, t)$ in terms of the kernel function $K(w, t)$ which is also a proof of the existence of $G(w, t)$. Our expansion formula will yield a number of the more important properties of the Green's function, and the construction will not refer explicitly to behavior on the boundary $C$ of $D$.

Our procedure is essentially an integration of (10). We consider the integral

$$
J(w, t)=\frac{1}{2 \pi} \iint_{D} \iint_{D} \frac{K(z, \zeta) \rho(z) \rho(\zeta) d \sigma(z) d \sigma(\zeta)}{(\bar{z}-\bar{w})(\zeta-t)} .
$$

By the orthogonality of the sine and cosine functions, it is clear that we can 
replace the factors $(\bar{z}-\bar{w})^{-1}$ and $(\zeta-t)^{-1}$ in the integrand by the bounded expressions

$$
\frac{\rho(z)-\rho(w)}{\rho(z)(\bar{z}-\bar{w})} \text { and } \frac{\rho(\zeta)-\rho(t)}{\rho(\zeta)(\zeta-t)}
$$

in circles about $z=w$ and $\zeta=t$, respectively, without altering the value of the integral. The new functions which we define in this manner are square integrable over $D$, and therefore we can expand $K(z, \zeta)$ in its series form (2) and integrate term by term at will. In particular, we find for $w \in B, t \in D$ :

$$
\begin{aligned}
J(w, t) & =\frac{1}{2 \pi} \iint_{D}\left\{\sum_{\nu=1}^{\infty} \overline{\phi_{\nu}(\zeta)} \iint_{D} \frac{\phi_{\nu}(z) \rho(z) d \sigma(z)}{\bar{z}-\bar{w}}\right\} \frac{\rho(\zeta) d \sigma(\zeta)}{\zeta-t} \\
& =\frac{1}{2 \pi} \iint_{D} \frac{\rho(\zeta) d \sigma(\zeta)}{(\bar{\zeta}-\bar{w})(\zeta-t)} .
\end{aligned}
$$

Now for fixed $t \in D$, one can show that the integral $J(w, t)$ is a continuous function of $w$ across $C$ in a manner essentially identical with that which led to the continuity relation ( 7$)$. Hence

$$
\lim _{w \rightarrow z \in C} J(w, t)=\frac{1}{2 \pi} \iint_{D} \frac{\rho(\zeta) d \sigma(\zeta)}{(\bar{\zeta}-\bar{z})(\zeta-t)}, \quad w, t \in D .
$$

Recalling the nature of a fundamental solution of (9), we now see that the Green's function $G(w, t)$ can be defined by the formula

$$
G(w, t)=\frac{1}{2 \pi} \iint_{D} \frac{\rho(\zeta) d \sigma(\zeta)}{(\bar{\zeta}-\bar{w})(\zeta-t)}-J(w, t) .
$$

Indeed, this expression satisfies (9) by Poisson's equation, and has zero boundary values by (18). We set

$$
b_{\nu}(w)=\iint_{D} \frac{\phi_{\nu}(z) \rho(z) d \sigma(z)}{(\bar{z}-\bar{w})}, \quad w \in D
$$

and we note that $b_{\nu}(w)$ is a particular solution of (9) in $D$. Thus (19) can be rewritten

$$
G(w, t)=\frac{1}{2 \pi} \iint_{D} \frac{\rho(\zeta) d \sigma(\zeta)}{(\bar{\zeta}-\bar{w})(\zeta-t)}-\frac{1}{2 \pi} \sum_{\nu=1}^{\infty} b_{\nu}(w) \overline{b_{\nu}(t)}
$$


and we have an expansion of the Green's function in terms of a symmetric geometric integral and a kernel function. We emphasize that the series of Fourier coefficients on the right converges uniformly in each closed subdomain of $D$ because of the square integrability of the modified functions involved. It is interesting in this connection that the Green's function represents according to (20) the difference between an improper geometrical integral and the development for this integral in terms of Fourier coefficients.

A first remark about formula (20) is that (10) follows from it by mere differentiation. The expression (20) is also significant in that it displays the positive definite character of the regular part of the Green's function, $J(w, t)$. Since $J(w, t)$ is expressed as a kernel function,

$$
J(w, t)=\frac{1}{2 \pi} \sum_{\nu=1}^{\infty} b_{\nu}(w) \overline{b_{\nu}(t)},
$$

many interesting inequalities are quite simply obtained. These are consequences of the positive nature of quantities of the type

$$
\begin{aligned}
|\lambda|^{2} J(w, w) & +\lambda \bar{\mu} J(w, t)+\bar{\lambda} \mu J(t, w)+|\mu|^{2} J(t, t) \\
& =\frac{1}{2 \pi} \sum_{\nu=1}^{\infty}\left|\lambda b_{\nu}(w)+\mu b_{\nu}(t)\right|^{2} \geq 0 .
\end{aligned}
$$

These inequalities have more or less the strength of the classical distortion theorem for schlicht functions. In fact, by substituting $\rho(z) \equiv 1$ and making suitable choice of the points $w$ and $t$ and the domain $D$, one can obtain by this procedure Koebe's original results. We note, finally, that formulas like (20) which represent the Green's function in terms of a geometrical integral and a kernel function are easily obtained in many further classes of functions; for example, such a formula $c$ an be obtained for the Green's function of the Laplace equation in three dimensions. These remarks tie in closelywith previous work on inequalities and geometrical integrals $[6,14]$.

The expansion (20) is new even in the simple case $\rho(z) \equiv 1$. Let us apply it to the calculation of $G(w, t)$ in the circular ring $r<|z|<1$ with $\rho(z) \equiv 1$. Here the set of functions $b_{\nu}(w)$ for (20) is found by Green's theorem to be

$$
\begin{aligned}
& b_{\nu}(w)=-\left(\frac{\pi}{(n+1)\left(1-r^{2 n+2}\right)}\right)^{1 / 5}\left(w^{n+1}+\frac{r^{2 n+2}}{\bar{w}^{n+1}}\right), n \neq-1, \\
& b_{2}(w)=-\left(\frac{-2}{\log r}\right)^{1 / 2} \log |w|,
\end{aligned}
$$


where we take $\nu=2 n+1, n \geq 0$, and $\nu=-2 n, n \leq-2$. Thus we obtain, for the Green's function $g(w, t)$ of the Laplace equation in the circular $\operatorname{ring} r<|z|<1$,

$$
\begin{aligned}
g(w, t) & =\frac{1}{2 \pi} \iint_{r<|z|<1} \frac{d \sigma(z)}{(\bar{z}-\bar{w})(z-t)}+\frac{(\log |w|)(\log |t|)}{\log r} \\
& -\sum_{n=-\infty, n \neq 0}^{\infty} \frac{1}{2 n\left(1-r^{2 n}\right)}\left(w^{n}+\frac{r^{2 n}}{\bar{w}^{n}}\right)\left(\bar{t}^{n}+\frac{r^{2 n}}{t^{n}}\right) .
\end{aligned}
$$

In closing this section, we note that for $\rho(z) \equiv 1, G(w, t)=g(w, t)$ is real, and therefore, from (20) and (10),

$k(t, w)=-\frac{2}{\pi} \frac{\partial^{2} g}{\partial \bar{w} \partial t}=\frac{1}{\pi^{2}} \sum_{\nu=1}^{\infty} \frac{\partial b_{\nu}}{\partial \bar{w}} \frac{\partial \overline{b_{\nu}}}{\partial t}-\frac{1}{\pi^{2}} \iint_{D} \frac{d \sigma}{((\bar{z}-\bar{w})(z-t))^{2}}$.

Hence, in this case, the kernel function has an interpretation in terms of the difference between the Cauchy principal value of an improper integral and the development of the integral in terms of Fourier coefficients.

5. An eigenvalue problem. We consider once more the kernel function $K(w, t)$ associated with a general weight function $\rho(z)$, and we study for a moment the integral equation

$$
\phi(t)=\lambda \iint_{D} K(t, z) \phi(z) d \sigma
$$

Let $\lambda_{\nu}$ be an eigenvalue and let $\phi_{\nu}(z)$ be the corresponding eigenfunction of this equation,

$$
\phi_{\nu}(t)=\lambda_{\nu} \iint_{D} K(t, z) \phi_{\nu}(z) d \sigma
$$

The scalar product of $\phi_{\nu}$ with any function $\phi \in \Omega$ is given by the interesting formula

$$
\begin{aligned}
\iint_{D} \overline{\phi_{\nu}(t)} \phi(t) \rho(t) d \sigma & =\lambda_{\nu} \iint_{D}\left[\iint_{D} K(z, t) \phi(t) \rho(t) d \sigma\right] \overline{\phi_{\nu}(z)} d \sigma \\
& =\lambda_{\nu} \iint_{D} \overline{\phi_{\nu}(z)} \phi(z) d \sigma .
\end{aligned}
$$

In particular, for $w \in B$ we have 


$$
\iint \frac{\phi_{\nu}(t)\left[\rho(t)-\lambda_{\nu}\right] d \sigma}{\bar{t}-\bar{w}}=0
$$

Thus, by the usual argument of continuity across $C$, the function

$$
F_{\nu}(w)=\iint_{D} \frac{\phi_{\nu}(t)\left[\rho(t)-\lambda_{\nu}\right] d \sigma}{\bar{t}-\bar{w}}, \quad w \in D
$$

has boundary values 0 on $C$, while in $D$ it satisfies the partial differential equation

$$
\frac{\partial}{\partial \bar{w}} \frac{1}{\rho(w)-\lambda_{\nu}} \frac{\partial}{\partial w} F_{\nu}(w)=0 .
$$

Thus the eigenfunctions of this non-linear eigenvalue problem in elliptic partial differential equations are connected with the eigenfunctions of the above integral equation,

$$
\frac{1}{\rho(w)-\lambda_{\nu}} \frac{\partial F_{\nu}(w)}{\partial w}=-\pi \phi_{\nu}(w)
$$

In the case of the circular ring $r<|z|<1$ with weight function $\rho=\rho(|z|)$ which depends only upon $|z|$, the powers of $z$ form a complete system of doubly orthogonal eigenfunctions of the type just described. Supposing that the $\phi_{\nu}(z)$ are normalized in the metric ( 1 ), we find

$$
K(w, t)=\sum_{\nu=1}^{\infty} \phi_{\nu}(w) \overline{\phi_{\nu}(t)},
$$

whereas for the kernel function $k(w, t)$ with weight function 1 , we have [2]

$$
K(w, t)=\sum_{\nu=1}^{\infty} \lambda_{\nu} \phi_{\nu}(w) \overline{\phi_{\nu}(t)} .
$$

However, the extremal problems

$$
\lambda=\frac{\iint_{D}|\phi(z)|^{2} \rho(z) d \sigma}{\iint_{D}|\phi(z)|^{2} d \sigma}=\text { maximum }
$$


and

$$
\lambda=\frac{\iint_{D}|\phi(z)|^{2} \rho(z) d \sigma}{\iint_{D}|\phi(z)|^{2} d \sigma}=\text { minimum }
$$

need not have solutions in $\Omega$, as can be seen, for example, by taking $\rho(z)=|z|^{2}$. Thus there is a difficulty, in general, in showing the existence of a complete system of eigenfunctions.

Note, in passing, that if we have two weight functions $\rho(z)$ and $\rho^{*}(z)$ in the domain $D$, the corresponding kernel functions $K(w, t)$ and $K^{*}(w, t)$ satisfy the integral equation

$$
K^{*}(w, t)=K(w, t)-\iint_{D} K(w, z) K^{*}(z, t)\left[\rho^{*}(z)-\rho(z)\right] d \sigma .
$$

Setting

$$
\begin{aligned}
\delta K(w, t) & =K^{*}(w, t)-K(w, t), \\
\delta \rho(z) & =\rho^{*}(z)-\rho(z)=O(\epsilon),
\end{aligned}
$$

we can write this as

$$
\delta K(w, t)=-\iint_{D} K(w, z) K(z, t) \delta \rho(z) d \sigma+O\left(\epsilon^{2}\right),
$$

a relation which is connected with the classical Hadamard variational formula [11] for certain classes of variations $\delta \rho(z)$ of our weight function.

We take $\rho(z) \equiv 1$, and we turn now to a somewhat different eigenvalue problem for which it is known that a complete orthonormal system of eigenfunctions $\psi_{\nu}(z)$ $\epsilon \Omega$ exists. The work of Friedrichs [7] gives us, indeed, a complete orthonormal system $\left\{\psi_{\nu}(z)\right\}$ satisfying

$$
\mu_{\nu} \iint_{D} \overline{\psi_{\nu}(z)} \phi(z) d \sigma=\iint_{D} \psi_{\nu}(z) \phi(z) d \sigma,
$$

with decreasing eigenvalues

$$
\mu_{1}=1>\mu_{2} \geq \mu_{3} \geq \cdots \geq 0
$$

which are obtained from the extremal problem

$$
\frac{\left|\iint_{D} \phi(z)^{2} d \sigma\right|}{\iint_{D}|\phi(z)|^{2} d \sigma}=\text { maximum. }
$$


We shall study (22) by the methods of this paper and shall associate with the eigenvalues $\mu_{\nu}$ of (22) a new eigenvalue problem for a related partial differential equation.

From (22) we have

$$
j=\iint_{D}\left\{\mu_{\nu} \overline{\psi_{\nu}(z)}-\psi_{\nu}(z)\right\} \frac{d \sigma}{z-w}=0, \quad w \in B .
$$

Thus, as usual, we define

$$
U_{\nu}(w)=\iint_{D}\left\{\mu_{\nu} \overline{\psi_{\nu}(z)}-\psi_{\nu}(z)\right\} \frac{d \sigma}{z-w}, \quad w \in D,
$$

and we show that $U_{\nu}(w)$ has boundary values zero on $C$ by proving that the integral $j$ is continuous across $C$. Let $\Gamma$ be a circle in $D$ tangent to $C$ at $w_{0}$, let $w$ be a point in $\Gamma$ on the normal to $C$ through $w_{0}$, and let $w^{*}$ be the inverse of $w$ in $\Gamma$. It suffices to show that

$$
\lim _{w \rightarrow w_{0}} \iint_{D}\left\{\mu_{\nu} \overline{\psi_{\nu}(z)}-\psi_{\nu}(z)\right\}\left\{\frac{1}{z-w}-\frac{1}{z-w^{*}}\right\} d \sigma=0 .
$$

This is true for the portion of the integral taken over $D-\Gamma$ by the square integrability of $\left|z-w_{0}\right|^{-1}$ there, as can be seen by comparison with the discussion in $\S 2$. For the integral over $\Gamma$, we see, again from $\$ 2$, that the contribution from the term $\mu_{\nu} \overline{\psi_{\nu}(z)}$ vanishes, and also that

$$
\iint_{\Gamma} \psi_{\nu}(w)\left\{\frac{1}{z-w}-\frac{1}{z-w^{*}}\right\} d \sigma=0 .
$$

We have thus from the Gauss mean value theorem

$$
\begin{aligned}
\iint_{\Gamma} \psi_{\nu}(z)\left\{\frac{1}{z-w}-\frac{1}{z-w^{*}}\right\} d \sigma & =\iint_{\Gamma}\left\{\psi_{\nu}(z)-\psi_{\nu}(w)\right\} \frac{\left(w-w^{*}\right) d \sigma}{(z-w)\left(z-w^{*}\right)} \\
& =\left[\psi_{\nu}\left(z_{0}\right)-\psi_{\nu}(w)\right] \frac{\left(w-w^{*}\right) \pi \eta^{2}}{\left(z_{0}-w\right)\left(z_{0}-w^{*}\right)},
\end{aligned}
$$

where $z_{0}$ is the center and $\eta$ the radius of $\Gamma$. Thus it remains only to show that

$$
\lim _{w \rightarrow w_{0}} \psi_{\nu}(w)\left(w-w^{*}\right)=0 \text {. }
$$

Let $\gamma(w)$ be the largest circle in $D$ centered at $w$, and let the radius of $\gamma(w)$ be $\epsilon$. Then $w-w^{*}=O(\epsilon)$ because of the smoothness of $C$; and the mean value 
theorem yields, for some $M>0$,

$$
\begin{aligned}
& \text { for some } M>0, \\
& \begin{aligned}
\left|\psi_{\nu}(w)^{2}\left(w-w^{*}\right)^{2}\right| & =\left|\frac{\left(w-w^{*}\right)^{2} \iint_{\gamma} \psi_{\nu}(z)^{2} d \sigma}{\pi \epsilon^{2}}\right| \\
& \leq M \iiint_{\gamma(w)}\left|\psi_{\nu}(z)^{2}\right| d \sigma .
\end{aligned}
\end{aligned}
$$

The last integral tends to zero as $w \longrightarrow w_{o}$ and $\epsilon \longrightarrow 0$, since $\psi_{\nu}(z)$ is square integrable, and hence our continuity proof is complete.

We now derive a partial differential equation of the second order for the function $U_{\nu}(w)$, which vanishes on $C$. By Poisson's equation we have

$$
\frac{\partial U_{\nu}}{\partial \bar{w}}=-\pi \mu_{\nu} \overline{\psi_{\nu}(w)}+\pi \psi_{\nu}(w)
$$

Taking conjugates, we find

$$
\frac{\partial \bar{U}_{\nu}}{\partial w}=-\pi \mu_{\nu} \psi_{\nu}(w)+\pi \overline{\psi_{\nu}(w)}
$$

and therefore

$$
\pi\left(1-\mu_{\nu}^{2}\right) \psi_{\nu}(w)=\frac{\partial U_{\nu}}{\partial \bar{w}}+\mu_{\nu} \frac{\partial \bar{U}_{\nu}}{\partial w}
$$

By the Cauchy-Riemann equations for $\psi_{\nu}(w)$ we obtain

$$
\frac{\partial^{2} U_{\nu}}{\partial \bar{w}^{2}}+\mu_{\nu} \frac{\partial^{2} \bar{U}_{\nu}}{\partial \bar{w} \partial w}=0 .
$$

Hence the functions $U_{\nu}(w)$ are the eigenfunctions and the $\mu_{\nu}$ are the eigenvalues for the eigenvalue problem

$$
\frac{\partial^{2} U}{\partial \bar{w}^{2}}+\mu \frac{\partial^{2} \bar{U}}{\partial \bar{w} \partial w}=0
$$

with $U \equiv 0$ on $C$.

For $\nu>1$ the eigenfunctions $\psi_{\nu}(w)$ are obtained from the $U_{\nu}(w)$ by means of formula (24). Green's theorem can then be applied to yield (22). Therefore (22) and (25) are quite equivalent eigenvalue problems. Friedrichs' principal result, the strong inequality $\mu_{2}<\mu_{1}=1$, has now a new significance, particularly since 
(24) cannot be solved for the first (constant) eigenfunction $\psi_{1}(w)$.

One sees, in general, from the results obtained so far, then, how problems in the study of analytic functions of class $\Omega$ in a domain $D$ lead naturally to the discussion of systems of two partial differential equations of the second order, such as (9) and (25). We remark, in closing, that the solutions $U(w)$ of (25) always satisfy, in particular, the single fourth order elasticity equation

$$
\frac{\partial^{4} U(w)}{\partial w^{2} \partial \bar{w}^{2}}=0
$$

6. Extensions. It is quite clear that the method which has led us here to results such as (10), (20), and (25) has wide applications to the study of the existence and representation of solutions of linear partial differential equations of elliptic type. Equations which can be discussed in this manner, such as the equation

$$
u_{x x}+u_{y y}+P(x, y) u=0, \quad P<0 \text {, }
$$

need have no connection with analytic functions. However, we shall consider in this section one more case which is drawn from the theory of harmonic functions.

We introduce the class $\Lambda$ of real-valued harmonic functions $u(z)$ in $L$ with a finite integral

$$
\iint_{D} u(z)^{2} \rho(z) d \sigma
$$

where $\rho(z)$ is once more a positive weight function with, say, continuous second derivatives in a region including $D+C$. By the Gauss mean value theorem and the Schwarz inequality there is a positive function $A(z)$ in $D$ such that

$$
\left|u\left(z_{0}\right)\right| \leq A\left(z_{0}\right)\left(\iint_{D} u(z)^{2} \rho(z) d \sigma\right)^{1 / 2}, \quad z_{0} \in D .
$$

Hence there exists in $\Lambda$ a complete orthonormal system $\left\{u_{\nu}(z)\right\}$,

$$
\iint_{D} u_{\nu}(z) u_{\mu}(z) \rho d \sigma=\delta_{\mu \nu}
$$

the kernel function

$$
M(z, t)=\sum_{\nu=1}^{\infty} u_{\nu}(z) u_{\nu}(t)
$$


converges and is in $\Lambda$, and we have the reproducing property

$$
u(t)=\iint_{D} M(t, z) u(z) \rho d \sigma,
$$

In particular, for $w \in B$, the exterior of $i$, we obtain

$$
\log \frac{1}{|t-w|}-\iint_{D} N(t, z) \log \frac{1}{|z-w|} \rho d \sigma=0 .
$$

lience with $w \in B, \zeta \in D$ we find

$$
\begin{aligned}
\iint_{D} \log & \frac{1}{|t-w|} \log \frac{1}{|t-\zeta|} \rho(t) d \sigma(t) \\
& \quad-\iint_{D} \iint_{D} M(z, t) \log \frac{1}{|z-w|} \log \frac{1}{|t-\zeta|} \rho(z) \rho(t) d \sigma(z) d \sigma(t)=0,
\end{aligned}
$$

or, more directly,

$$
\begin{aligned}
\iint_{D} \log \frac{1}{|t-w|} \log \frac{1}{|t-\zeta|} \rho d \sigma-\sum_{\nu=1}^{\infty} V_{\nu}(w) V_{\nu}(\zeta) & =0, \\
& w \in E, \quad \zeta \in D,
\end{aligned}
$$

where

$$
V_{\nu}(w)=\iint_{D} u(z) \log \frac{1}{|z-w|} \rho(z) d \sigma(z), \quad \nu=1,2,3, \cdots,
$$

are the Fourier coefficients of the function $-\log |z-w|$ for $w \in B$, and also for $w \in D$. The statement is, then, that the integral

$$
\iint_{D} \log \frac{1}{|t-w|} \log \frac{1}{|t-\zeta|} \rho d \sigma
$$

is represented faithfully by its development in terms of Fourier coefficients with respect to the orthonormal system $\left\{u_{\nu}\right\}$ when $w \in B$, because in this case the first factor $-\log |t-w|$ in the integrand belongs to the class $\Lambda$, and the second factor $-\log |t-\zeta|$ is square integrable.

When both $\zeta$ and $w$ are in $D$, we set

$$
H(w, \zeta)=\frac{2}{\pi} \iint_{D} \log \frac{1}{|t-w|} \log \frac{1}{|t-\zeta|} \rho d \sigma-\frac{2}{\pi} \sum_{\nu=1}^{\infty} V_{\nu}(w) V_{\nu}(\zeta)
$$


and we proceed to show that $H(w, \zeta)$ is the Green's function of the fourth order elliptic partial differential equation

$$
\Delta \frac{1}{\rho} \Delta h=0, \quad \Delta=4 \frac{\partial^{2}}{\partial z \partial \bar{z}} .
$$

Indeed, from the square integrability of $-\log |z-w|$ and Schwarz's inequality, together with Bessel's inequality

$$
\sum_{\nu=1}^{\infty} V_{\nu}(\zeta)^{2} \leq \iint_{D}\left\{\log \frac{1}{|t-\zeta|}\right\}^{2} \rho d \sigma<\infty
$$

it is apparent that the kernel series

$$
\sum_{\nu=1}^{\infty} V_{\nu}(w) V_{\nu}(\zeta)
$$

is continuous across $C$, and therefore by comparison with (28) we find

$$
\lim _{w \rightarrow C} H(w, \zeta)=0,
$$

It is more difficult, however, to verify the requirement

$$
\frac{\partial H(w, \zeta)}{\partial \nu}=0
$$

for the derivative of $H$ with respect to the inner normal $\nu$ of $C$. We shall prove this condition by showing that the gradient

$$
2 \frac{\partial l i}{\partial w}=\frac{2}{\pi} \iint_{D} \frac{1}{z-w}\left\{\log \frac{1}{|z-\zeta|}-\sum_{\nu=1}^{\infty} V_{\nu}(\zeta) u_{\nu}(z)\right\} \rho(z) d \sigma
$$

tends to zero as $w \rightarrow C$.

By comparison with (28), we see that it is sufficient to show that

$$
\lim _{w \rightarrow C} \iint_{D}\left\{\frac{1}{z-w}-\frac{1}{z-w^{*}}\right\} V(z) \rho(z) d \sigma=0,
$$

where $V(z)$ represents the terms previously in brackets, and where $w$ and $w^{*}$ lie on the normal to $C$ through a point $w_{0}$ and are inverse points in a circle $\Gamma$ in $D$ 
tangent to $C$ at $w_{0}$. Since

$$
\begin{gathered}
\frac{1}{z-w}-\frac{1}{z-w^{*}}=O\left(\frac{1}{\left|z-w_{0}\right|}\right), \quad z \in D-\Gamma, \\
\left\{\frac{1}{z-w}-\frac{1}{z-w^{*}}\right\}[\rho(z)-\rho(w)]=O(1), \quad z \in \Gamma,
\end{gathered}
$$

and since these dominants are square integrable over $D-\Gamma$ and $\Gamma$, respectively, we see, as in previous continuity proofs, that the Lebesgue theorem applies to the part of our integral extended over $D-\Gamma$ and that it applies to the part extended over $\Gamma$ with $\rho(z)$ replaced by $\rho(z)-\rho(w)$. Thus the only difficulty is to prove

$$
\lim _{w \rightarrow w_{0}} \iint_{\Gamma}\left\{\frac{1}{z-w}-\frac{1}{z-w^{*}}\right\} V(z) d \sigma=0 .
$$

Now in the circle $\Gamma, V(z)$ has a harmonic conjugate $V^{*}(z)$ which is also square integrable, and hence the analytic function

$$
W(z)=V(z)+i V^{*}(z)
$$

is square integrable over $\Gamma$. By (6) we have

$$
\iint_{\Gamma}\left\{\frac{1}{z-w}-\frac{1}{z-w^{*}}\right\} \overline{W(z)} d \sigma=0
$$

and also

$$
\iint_{\Gamma}\left\{\frac{1}{z-w}-\frac{1}{z-w^{*}}\right\} W(w) d \sigma(z)=0 .
$$

Therefore

$$
\begin{aligned}
\iint_{\Gamma}\left\{\frac{1}{z-w}-\frac{1}{z-w^{*}}\right\} V(z) d \sigma & =\frac{1}{2} \iint_{\Gamma}\left\{\frac{1}{z-w}-\frac{1}{z-w^{*}}\right\}[W(z)+\overline{W(z)}] d \sigma \\
& =\frac{1}{2} \iint_{\Gamma}\left\{\frac{1}{z-w}-\frac{1}{z-w^{*}}\right\}[W(z)-W(w)] d \sigma \\
& =\frac{\pi \eta^{2}}{2} \frac{\left[W\left(z_{0}\right)-W(w)\right]\left(w-w^{*}\right)}{\left(z_{0}-w\right)\left(z_{0}-w^{*}\right)}
\end{aligned}
$$


by the Gauss mean value theorem, where $z_{0}$ is the center and $\eta$ the radius of $\Gamma$. Letting $\gamma(w)$ be the largest circle in $\Gamma$ centered at $z=w$, we obtain for some $\|>0$, again by the mean value theorem,

$$
\left|W(w)^{2}\left(w-w^{*}\right)^{2}\right| \leq M \iiint_{\gamma(w)}|W(z)|^{2} d \sigma
$$

and since $\mathbb{W}(z)$ is square integrable, the last integral tends to zero as $w \longrightarrow w_{0}$. This completes the proof of (31).

Thus we have, on $C$,

$$
H(w, \zeta)=\frac{\partial H(w, \zeta)}{\partial \nu}=0
$$

By application of Poisson's equation and term by term differentiation, we verify immediately that $H(w, \zeta)$ satisfies $(30)$, and it is furthermore clear that the integral in formula (29) is a fundamental solution of (30). Hence $H(w, \zeta)$ is, indeed, the Green's function of (30). We now obtain by differentiation of (29) and two applications of Poisson's equation the formula

$$
M(w, \zeta)=-\frac{1}{8 \pi \rho(w) \rho(\zeta)} \quad \Delta_{w} \Delta_{\zeta} H(w, \zeta)
$$

expressing the kernel function in terms of the Green's function. The usual deductions as to the positive definite nature [11] of $H(w, \zeta)$ follow from (29) and (33).

The significance of the norm (26) for the equation (30) is apparent from the form

$$
\iint_{D}(\Delta h)^{2} \frac{1}{\rho} d \sigma
$$

of the Euler integral of (30) and the form

$$
h(w)=\iint_{D} u_{1}(z) \rho(z) \log \frac{1}{|z-w|} d \sigma+u_{2}(w)
$$

of the general solution of (30), expressed in terms of two arbitrary harmonic functions. We have, thus,

$$
\iint_{D}(\Delta h)^{2} \frac{1}{\rho} d \sigma=4 \pi^{2} \iint_{D} u_{1}^{2} \rho d \sigma
$$

It is interesting that we obtain the existence of the Green's function $H(w, \zeta)$ using a norm which depends on only half the number of arbitrary functions involved in 
the general solution of (30). Also, the connection between extremal functions for the norm (26) and solutions of (30) with simple boundary behavior can be explained by use of the Green's identity

$$
\iint\{p \Delta q-q \Delta p\} d \sigma+\oint\left\{p \frac{\partial q}{\partial \nu}-q \frac{\partial p}{\partial \nu}\right\} d s=0,
$$

in which we can take, for example, $p \in \Lambda$ and $q$ a solution of (30).

We point out that for $\rho(z) \equiv 1$, equation (30) is merely the equation of an elastic plate. Thus our method leads to a new $[17,18]$ discussion of the existence and representation of the solutions of boundary value problems in elasticity. This case has an interesting connection with Friedrichs' eigenfunctions $\left\{\psi_{\nu}(z)\right\}$, discussed in $\$ 5$. Indeed, setting

$$
\psi_{\nu}(z)=u_{\nu}(z)+i v_{\nu}(z),
$$

we obtain, from (22),

$$
\begin{gathered}
\iint_{D}\left\{u_{\nu} u_{\mu}+v_{\nu} v_{\mu}+i u_{\nu} v_{\mu}-i u_{\mu} v_{\nu}\right\} d \sigma=\delta_{\mu \nu}, \\
\iint_{D}\left\{u_{\nu} u_{\mu}-v_{\nu} v_{\mu}+i u_{\nu} v_{\mu}+i u_{\mu} v_{\nu}\right\} d \sigma=\mu_{\nu} \delta_{\mu \nu} .
\end{gathered}
$$

Thus for a simply connected domain $D$, in which each harmonic function $u(z)$ has a single-valued conjugate $v(z)$, the system of functions

$$
\begin{array}{ll}
\left(\frac{2}{1+\mu_{\nu}}\right)^{1 / 2} u_{\nu}(z), & \nu=1,2, \cdots, \\
\left(\frac{2}{1-\mu_{\nu}}\right)^{1 / 2} v_{\nu}(z), & \nu=2,3, \cdots,
\end{array}
$$

form a complete orthonormal system in $\Lambda$. Therefore, with $\rho(z) \equiv 1$, we have

$$
M(z, t)=2 \sum_{\nu=1}^{\infty} \frac{u_{\nu}(z) u_{\nu}(t)}{1+\mu_{\nu}}+2 \sum_{\nu=2}^{\infty} \frac{v_{\nu}(z) v_{\nu}(t)}{1-\mu_{\nu}},
$$

while

$$
k(z, t)=\sum_{\nu=1}^{\infty}\left\{u_{\nu}(z)+i v_{\nu}(z)\right\}\left\{u_{\nu}(t)-i v_{\nu}(t)\right\} .
$$


Hence the orthonormal eigenfunctions $\left\{\psi_{\nu}(z)\right\}$ and the corresponding eigenvalues $\mu_{\nu}$ provide a link between the analytic functions of class $\Omega$ and the harmonic functions of class $\Lambda$ which arise in elasticity.

In particular, for the interior of an ellipse the $\psi_{\nu}(z)$ are the Chebyshev polynomials of second' kind [7] and the Green's function $H(z, t)$ has an explicit representation (29). Suppose, in fact, that $l$ is the ellipse

$$
\frac{x^{2}}{\cosh ^{2} \sigma}+\frac{y^{2}}{\sinh ^{2} \sigma}<1
$$

so that

$$
\psi_{\nu}(z)=\rho_{\nu} \frac{\sinh \left(\nu \cosh ^{-1} z\right)}{\sinh \left(\cosh ^{-1} z\right)}, \mu_{\nu}=\frac{\rho_{\nu}^{2}}{\rho_{1}^{2}}, \rho_{\nu}=\left(\frac{2 \nu}{\pi \sinh 2: \sigma}\right)^{1 / 2},
$$

Then we obtain the formula of Erdélyi and Zaremba:

$$
\nu=1,2, \cdots
$$

$$
\begin{aligned}
H(z, t) & =\frac{2}{\pi} \iint_{D} \log \frac{1}{|z-w|} \log \frac{1}{|t-w|} d \sigma \\
& -\frac{4 \rho^{2}}{\pi} \sum_{\nu=1}^{\infty} \frac{\rho \nu^{2}}{\rho_{1}^{2}+\rho_{\nu}^{2}}\left\{\iint_{D} \log \frac{1}{|z-w|} \Re\left\{\frac{\sinh \left(\nu \cosh ^{-1} z\right)}{\sinh \left(\cosh ^{-1} z\right)}\right\} d \sigma\right. \\
& -\frac{4 \rho^{2}}{\pi} \sum_{\nu=2}^{\infty} \frac{\rho_{\nu}^{2}}{\rho_{1}^{2}-\rho_{\nu}^{2}}\left\{\iint_{D} \log \frac{1}{|t-\zeta|} \Re\left\{\frac{\sinh \left(\nu \cosh ^{-1} z\right)}{\sinh \left(\cosh ^{-1} z\right)}\right\} d \sigma\right\} \\
& \left\{\frac{1}{|z-w|}\left\{\frac{\sinh \left(\nu \cosh ^{-1} z\right)}{\sinh \left(\cosh ^{-1} z\right)}\right\} d \sigma\right.
\end{aligned}
$$

The integrals can be reduced by using Green's theorem.

This tie between the elasticity equation and the Laplace equation by means of the Friedrichs eigenfunctions can be generalized to the case of multiply connected domains. Here, however, we must restrict the class $\Lambda$ to functions $u(z)$ with single-valued conjugates $v(z)$ and we must replace $H(z, t)$ by a similar function 
which has merely constant boundary values on each component of $C$ and zero normal derivatives.

Finally, the reader will notice that the case where $\rho(z)=|\mu(z)|^{2}$, the square of the modulus of an analytic function $\mu(z)$, also has bearing on the elasticity equation. Here, in fact, the general solution of the equation (30) is

$$
h(z)=\mathfrak{R}\{\overline{M(z)} f(z)+g(z)\},
$$

where $M^{\prime}(z)=\mu(z)$ and $f(z), g(z)$ are analytic. Thus the conformal transformation $\zeta=M(z)$ reduces $(30)$ to the elasticity equation.

7. A numerical example. We add at this point a note concerning the nature of the deflection of the clamped elliptical plate of $\$ 6$. It has been conjectured by Hadamard [11] that the Green's function $H(z, t)$ of the elasticity equation in a domain $D$ is positive. This conjecture has the interpretation that when one places a downward point load at $t \in D$ upon an infinitesimally thin elastic plate clamped at $C$, the resulting deflection $H(z, t)$ at $z \in D$ is always directed downward, with the load. Duffin has shown that $H(z, t)$ can become negative when $D$ is an infinite strip, while Loewner and Szegö have exhibited bounded regions for which the conjecture is untrue, although these regions are not convex. The statement is true for a circle.

We discuss here an ellipse for which the Green's function can take negative values, and we thus obtain a counterexample even for the case of bounded convex plates, where the conjecture of Hadamard appears to be most plausible. Our procedure provides an illustration of the adaptability of the method of orthogonal functions to computational problems.

Our first remark is that if $H(z, t)$ is nonnegative in a domain $D$, then the fourth derivative

$$
\frac{\partial^{4} H(z, t)}{\partial n^{2}(z) \partial n^{2}(t)}
$$$$
z, t \in C,
$$

must also be nonnegative, where $n(z)$ and $n(t)$ represent the inner normals at $z$ and $t$ on $C$. This result follows immediately from a consideration of the Taylor's series for $H(w, \zeta)$ about $w=z, \zeta=t$. By the boundary conditions imposed upon $H(z, t)$, the second derivatives of $H(z, t)$ with respect to the arc lengths $s(z)$ and $s(t)$ at $z$, $t \in C$ vanish. Thus we obtain

$$
\frac{\partial^{4} H(z, t)}{\partial n^{2}(z) \partial n^{2}(t)}=\Delta_{z} \Delta_{t} H(z, t), \quad z, t \in C,
$$


and therefore, by (33),

$$
\frac{\partial^{4} H(z, t)}{\partial n^{2}(z) \partial n^{2}(t)}=-8 \pi M(z, t), \quad z, t \in C .
$$

Thus we can show that Hadamard's conjecture is false by exhibiting points $z$ and $t$ on $C$ for which the kernel function $h(z, t)$ is positive.

Let now $D$ be the ellipse

$$
\frac{x^{2}}{\cosh ^{2} \sigma}+\frac{y^{2}}{\sinh ^{2} \sigma}<1
$$

of $\S 6$, and take for $z, t \in C$ the vertices

$$
z=\cosh \sigma, \quad t=-\cosh \sigma .
$$

Then, by $\$ 6$,

$$
\begin{aligned}
M(z, t) & =\lim _{\substack{x \rightarrow \cosh \sigma \\
x<\cosh \sigma}} 2 \rho_{1}^{2} \sum_{\nu=1}^{\infty} \frac{\rho_{\nu}^{2}}{\rho_{1}^{2}+\rho_{\nu}^{2}} \frac{\sinh \left(\nu \cosh ^{-1} x\right)}{\sinh \left(\cosh ^{-1} x\right)} \frac{\sinh \left(\nu \cosh ^{-1}(-x)\right)}{\sinh \left(\cosh ^{-1}(-x)\right)} \\
& =\lim _{r \rightarrow \sigma, r<\sigma} 2 \rho_{1}^{2} \sum_{\nu=1}^{\infty}(-1)^{\nu+1} \frac{(\nu \sinh 2 \sigma)}{\sinh 2 \nu \sigma+\nu \sinh 2 \sigma} \frac{\sinh ^{2} \nu r}{\sinh ^{2} r}
\end{aligned}
$$

Thus it suffices to prove for some $\sigma>0$ that

$$
\lim _{r \rightarrow \sigma, r<\sigma} \sum_{\nu=1}^{\infty}(-1)^{\nu+1} \frac{\nu \sinh ^{2} \nu r}{\sinh 2 \nu \sigma+\nu \sinh 2 \sigma}>0 .
$$

We note that

$$
\lim _{r \rightarrow \sigma} \sum_{\nu=1}^{\infty}(-1)^{\nu+1} \frac{\nu e^{2 \nu r}}{e^{2 \nu \sigma}}=\frac{1}{4}
$$

and therefore we can replace (35) by

$$
\sum_{\nu=1}^{\infty}(-1)^{\nu+1}\left\{\frac{2 \nu \sinh ^{2} \nu \sigma}{\sinh 2 \nu \sigma+\nu \sinh 2 \sigma}-\nu\right\}+\frac{1}{4}>0 .
$$


Wie set $\sigma=\log 2$, and this becomes

$$
\sum_{\nu=1}^{\infty}(-1)^{\nu} \frac{2 \nu-2 \nu 2^{-2 \nu}+\nu^{2}\left(2^{2}-2^{-2}\right)}{2^{2 \nu}-2^{-2 \nu}+\nu\left(2^{2}-2^{-2}\right)}+\frac{1}{4}>0,
$$

an inequality which can be verified by direct calculation.

Briefly, the first ten terms of the series (36) are

$$
\begin{array}{cccccc}
-.7, \quad .8,-.527103, & .250811, & -.099487, & .035692, & -.012050, \\
& .003904,-.001227, \quad .000377,
\end{array}
$$

and we have

$$
\sum_{\nu=1}^{\infty}(-1)^{\nu} \frac{2 \nu-2 \nu 2^{-2 \nu}+\nu^{2}\left(2^{2}-2^{-2}\right)}{2^{2 \nu}-2^{-2 \nu}+\nu\left(2-2^{-2}\right)}+\frac{1}{4} \geq \frac{1}{2000} .
$$

Thus the Green's function $H(z, t)$ of an ellipse whose major axis is not even twice as long as its minor axis takes negative values when the arguments $z$ and $t$ are sufficiently near the vertices.

Finally, keeping $z=\cosh \sigma$, but setting $t=i \sinh \sigma$, we have

$$
\begin{aligned}
M(z, t) & =\lim _{r \rightarrow \sigma, r<\sigma} 2 \rho_{1}{ }^{2} \sum_{\nu=1}^{\infty}(-1)^{\nu+1} \frac{(2 \nu-1)(\sinh 2 \sigma)}{\sinh (4 \nu-2) \sigma+(2 \nu-1) \sinh 2 \sigma} \\
& =2 \rho_{1}^{2} \sum_{\nu=1}^{\infty}(-1)^{\nu}\left\{\frac{\sinh (2 \nu-1) r}{\sinh r} \frac{\cosh (2 \nu-1) r}{\cosh r}\right.
\end{aligned}
$$

Therefore to obtain $M(z, t)>0$ in this case, we take $\sigma=(1 / 2) \log 2$ and prove the elementary inequality

$$
\sum_{\nu=1}^{\infty}(-1)^{\nu} \frac{(2 \nu-1)^{2}}{2^{2 \nu-1}-2^{-2 \nu+1}+(2 \nu-1)\left(2-2^{-1}\right)}>0 .
$$

Indeed, the first ten terms of this series are 
A PARTIAL DIFFERENTIAL EQUATION ARISING IN CONFORMAL MAPPING

$$
\begin{array}{cccccc}
-.333333, & .727272, & -.633413, & .353811, & -.154139, & .058610, \\
-.020580, & .006862, & -.002204, & .000688, &
\end{array}
$$

and

$$
\sum_{\nu=1}^{\infty}(-1)^{\nu} \frac{(2 \nu-1)^{2}}{2^{2 \nu-1}-2^{-2 \nu+1}+(2 \nu-1)\left(2-2^{-1}\right)} \geq \frac{3}{1000} .
$$

8. On Friedrichs' functions. Bergman and Schiffer [4] have introduced the class $\mathfrak{M}$ of biharmonic functions $\bar{z} f(z)+g(z)$ in $D$ with a finite square integral

$$
\iint_{D}|\bar{z} f(z)+g(z)|^{2} d \sigma .
$$

They construct from a complete orthonormal system $\left\{\bar{z} f_{\nu}(z)+g_{\nu}(z)\right\}$ in $\mathbb{M}$,

$$
\iint_{D}\left[\bar{z} f_{\nu}(z)+g_{\nu}(z)\right]\left[z \overline{f_{\mu}(z)}+\overline{g_{\mu}(z)}\right] d \sigma=\delta_{\nu \mu},
$$

the kernel function

$$
N(z, t)=\sum_{\nu=1}^{\infty}\left\{\bar{z} f_{\nu}(z)+g_{\nu}(z)\right\}\left\{t \overline{t f_{\nu}(t)}+\overline{g_{\nu}(t)}\right\},
$$

and prove that this kernel is given by

$$
N(z, t)=-\frac{2}{\pi} \frac{\partial^{4} H(z, t)}{\partial z^{2} \partial \bar{t}^{2}},
$$

where $H(z, t)$ is, again, the Green's function of the elasticity equation. We leave to the reader the derivation of the inverse formula

$$
\begin{aligned}
H(z, t)= & \frac{1}{2 \pi} \iint_{D} \frac{z-w}{\bar{z}-\bar{w}} \frac{\bar{t}-\bar{w}}{t-w} d \sigma(w) \\
& -\frac{1}{2 \pi} \iiint_{D} \iint_{D} \frac{z-w}{\bar{z}-\bar{w}} \frac{\bar{t}-\bar{\zeta}}{t-\zeta} N(w, \zeta) d \sigma(\mathrm{w}) d \sigma(\zeta),
\end{aligned}
$$

which is based on considerations similar to those leading up to (20) and (29). Likewise, we leave to the reader a discussion, to be modeled on $\$ 2$, of the kernel 
$N_{\rho}(z, t)$ of the norm

$$
\iint_{D}|\bar{z} f(z)+g(z)|^{2} \rho(z) d \sigma
$$

with weight $\rho$; the kernel is given by the relation

$$
N_{\rho}(z, t)=-\frac{2}{\pi \rho(z) \rho(t)} \frac{\partial^{4} H_{\rho}(z, t)}{\partial z^{2} \partial \bar{t}^{2}},
$$

where $H_{\rho}(z, t)$ is the Green's function of the equation

$$
\frac{\partial^{2}}{\partial \overline{\mathbf{z}}^{2}} \frac{1}{\rho} \frac{\partial^{2}}{\partial z^{2}} u=0
$$

with energy integral

$$
\iint_{D} \frac{1}{\rho}\left|\frac{\partial^{2} u}{\partial z^{2}}\right|^{2} d \sigma .
$$

We show here how the kernel $N(z, t)$ can be constructed in terms of Friedrichs' eigenfunctions $\left\{\psi_{\nu}(z)\right\}$. In $\S 5$, we introduced the associated eigenfunctions

$$
U_{\nu}(w)=\iint_{D}\left\{\mu_{\nu} \overline{\psi_{\nu}(z)}-\psi_{\nu}(z)\right\} \frac{d \sigma}{z-w}, \quad w \in D .
$$

We have, by (22),

$$
\begin{aligned}
\iint_{D} \frac{\partial U_{k}}{\partial \bar{w}} \frac{\partial \bar{U}_{l}}{\partial w} d \sigma= & \pi^{2} \mu_{k} \mu_{l} \iint_{D} \bar{\psi}_{k} \psi_{l} d \sigma-\pi^{2} \mu_{l} \iint_{D} \psi_{k} \psi_{l} d \sigma \\
& -\pi^{2} \mu_{k} \iint_{D} \bar{\psi}_{k} \bar{\psi}_{l} d \sigma+\pi^{2} \iint_{D} \psi_{k} \bar{\psi}_{l} d \sigma \\
= & \pi^{2}\left\{1-\mu_{k}^{2}\right\} \delta_{k l} .
\end{aligned}
$$

But since $U_{k}(z) \equiv 0$ for $z \in C$, we can apply Green's theorem to obtain

$$
\begin{aligned}
\int_{D} \frac{\partial U_{k}}{\partial w} \frac{\partial \bar{U}_{l}}{\partial \bar{w}} d \sigma & =-\iint_{D} U_{k} \frac{\partial^{2} \bar{U}_{l}}{\partial w \partial \bar{w}} d \sigma \\
& =\iint_{D} \frac{\partial U_{k}}{\partial \bar{w}} \frac{\partial \bar{U}_{l}}{\partial w} d \sigma=\pi^{2}\left\{1-\mu_{k}^{2}\right\} \delta_{k l},
\end{aligned}
$$

and thus the system of biharmonic functions

$$
\left\{\frac{1}{\pi\left(1-\mu_{k}^{2}\right)^{1 / 2}} \frac{\partial U_{k}(w)}{\partial w}\right\} \quad, \quad k=2,3, \cdots
$$


is orthogonal in the class $\mathbb{M}$. Note that

$$
\frac{\partial U_{k}(w)}{\partial w}=\pi \bar{w} \psi_{k}^{\prime}(w)+\pi F_{k}^{\prime}(w)
$$

with analytic $F_{k}(w)$, and

$$
\frac{\partial^{3} U_{k}}{\partial w \partial \bar{w}^{2}}=0
$$

Furthermore, we have once more from Green's theorem

$$
\iint_{D} \frac{\partial U_{k}}{\partial w} \overline{\phi(w)} d \sigma=-\iint_{D} U_{k}(w) \frac{\partial \bar{\phi}}{\partial w} d \sigma=0
$$

for each analytic function $\phi \in \Omega$. Thus for simply connected regions $D$ the functions $\partial U_{k} / \partial w$ generate the subclass of biharmonic functions in $M$ which are orthogonal to the analytic functions of class $\Omega$.

We now recognize that for a simply connected domain $D$ the system

$$
\left\{\frac{1}{\left(1-\mu_{k}^{2}\right)^{1 / 2}} \frac{\partial U_{k}(w)}{\partial w}\right\}+\left\{\psi_{l}(w)\right\}
$$

is complete and orthonormal in $\mathbb{M}$. We have therefore

$$
\begin{aligned}
N(z, t) & =\sum_{k=1}^{\infty} \dot{\psi}_{k}(z) \overline{\psi_{k}(t)}+\frac{1}{\pi^{2}} \sum_{k=2}^{\infty} \frac{1}{1-\mu_{k}^{2}} \frac{\partial U_{k}(z)}{\partial z} \frac{\partial \overline{U_{k}(t)}}{\partial \bar{t}} \\
& =k(z, t)+\frac{1}{\pi^{2}} \sum_{k=2}^{\infty} \frac{1}{1-\mu_{k}^{2}} \frac{\partial U_{k}(z)}{\partial z} \frac{\partial \overline{U_{k}(t)}}{\partial \bar{t}}
\end{aligned}
$$

where $k(z, t)$ is the kernel of the class $\Omega$ with weight function $\rho(z) \equiv 1$. This can also be written, according to (10) and (37),

(39) $-\frac{2}{\pi} \frac{\partial^{4} H(z, t)}{\partial z^{2} \partial \bar{t}^{2}}=-\frac{2}{\pi} \frac{\partial^{2} g(z, t)}{\partial z \partial \bar{t}}+\frac{1}{\pi^{2}} \sum_{k=2}^{\infty} \frac{1}{1-\mu_{k}^{2}} \frac{\partial U_{k}(z)}{\partial z} \frac{\partial \overline{U_{k}(t)}}{\partial \bar{t}}$.

Formula (39) can be integrated with respect to $z$ and $\bar{t}$ in such a manner that each term has zero boundary values on $C$, and thus

$$
\frac{\partial^{2} H(z, t)}{\partial z \partial \bar{t}}=g(z, t)-\frac{1}{2 \pi} \sum_{k=2}^{\infty} \frac{U_{k}(z) \overline{U_{k}(t)}}{1-\mu_{k}^{2}} .
$$


We have here an interesting relation between the biharmonic eigenfunctions $U_{k}$ and the Green's functions $g$ and $H$ of the Laplace and elasticity equations.

One consequence of (40) is found when we apply Schwarz's inequality to the kernel series on the right, thus obtaining inequalities between $g$ and $H$ (cf. $\S 4$ ). The simplest inequality of this form,

$$
\left.\left\{g(z, t)-\frac{\partial^{2} H(z, t)}{\partial z \partial \bar{t}}\right\}\right|_{z=t} \geq 0,
$$

comes from setting $z=t$ and yields an estimate of the logarithmic capacity in terms of the biharmonic Green's function $H(z, t)$. Furthermore, if we differentiate (40) with respect to $\bar{z}$ and $t$, we find

$$
M(z, t)=k(t, z)+\frac{1}{\pi^{2}} \sum_{k=2}^{\infty} \frac{1}{1-\mu_{k}^{2}} \frac{\partial U_{k}}{\partial \bar{z}} \frac{\partial \bar{U}_{k}}{\partial t},
$$

a formula equivalent to (34). Thus it is apparent that (40) is the most fundamental formula of this type so far developed.

These relations are easily generalized to the case of multiply connected regions $D$ by introducing a modified biharmonic Green's function $H(z, t)$ with zero normal derivatives, but with boundary values which are merely constant on each component of $C$. We are led to adjoin to our previous orthonormal system for $M$ a set of (orthonormalized) second derivatives $\partial^{2} W / \partial z^{2}$ of biharmonic functions $W(z)$ with zero normal derivatives and constant boundary values on each component of $C$. For higher connectivity, the functions $W$ play the role of generalized harmonic measures.

We turn next to the discussion of our results when $D$ is the ellipse

$$
\frac{x^{2}}{\cosh ^{2} \sigma}+\frac{y^{2}}{\sinh ^{2} \sigma}<1
$$

of $\$ \$ 6$ and 7. In this case the $\psi_{k}(z)$ are given in terms of the Chebyshev polynomials

$$
T_{k}(z)=\frac{\zeta^{k}+\zeta^{-k}}{2}, z=\frac{\zeta+\zeta^{-1}}{2},
$$

by the relations

$$
\psi_{k}(z)=\frac{\rho_{k}}{k} T_{k}^{\prime}(z)=\rho_{k} \frac{\zeta^{k}-\zeta^{-k}}{\zeta-\zeta^{-1}}, \quad \rho_{k}=\left(\frac{2 k}{\pi \sinh 2 k \sigma}\right)^{1 / 2} .
$$


From the general formula

$$
U_{k}(z)=-\pi \mu_{k} \int \overline{\psi_{k}(z)} d \bar{z}+\pi \bar{z} \psi_{k}(z)+\pi F_{k}(z),
$$

with $F_{k}(z)$ analytic but unknown, we verify that in the ellinse we have

$$
\begin{aligned}
U_{k}(z)=\pi \rho_{k} & \left\{-\frac{\sinh 2 \sigma}{\sinh 2 k \sigma} T_{k}(\bar{z})+\frac{\bar{z}}{k} T_{k}^{\prime}(z)\right. \\
& \left.-\frac{\sinh (2 k-2) \sigma}{\sinh 2 k \sigma} T_{k}(z)-\frac{\cosh 2 \sigma}{k-1} T_{k-1}^{\prime}(z)\right\} .
\end{aligned}
$$

Indeed, this expression vanishes identically on the boundary $C$ of the ellipse. We have discovered, then, a new orthogonality property of the combination

$$
\frac{1}{\pi \rho_{k}} \frac{\partial U_{k}}{\partial z}=\frac{\bar{z}}{k} T_{k}^{\prime \prime}(z)-\frac{\sinh (2 k-2) \sigma}{\sinh 2 k \sigma} T_{k}^{\prime}(z)-\frac{\cosh 2 \sigma}{k-1} T_{k-1}^{\prime \prime}(z)
$$

of Chebyshev polynomials in $\mathbb{M}$. We now rewrite (40) here to obtain

$$
\begin{aligned}
& \frac{\partial^{2} H(z, t)}{\partial z \partial \bar{t}}=g(z, t)-\sum_{k=2}^{\infty} \frac{k \operatorname{sh} 2 k \sigma}{\operatorname{sh}^{2} 2 k \sigma-k^{2} \operatorname{sh}^{2} 2 \sigma}\left\{-\frac{\operatorname{sh} 2 \sigma \operatorname{ch}^{2}\left(k \operatorname{ch}^{-1} \bar{z}\right)}{\operatorname{sh} 2 k \sigma}\right. \\
& +\bar{z} \frac{\operatorname{sh}\left(k \operatorname{ch}^{-1} z\right)}{\operatorname{sh}\left(\operatorname{ch}^{-1} z\right)}-\frac{\operatorname{sh}(2 k-2) \sigma}{\operatorname{sh} 2 k \sigma} \operatorname{ch}\left(k \operatorname{ch}^{-1} z\right) \\
& \left.-\operatorname{ch} 2 \sigma \frac{\operatorname{sh}\left((k-1) \operatorname{ch}^{-1} z\right)}{\operatorname{sh}\left(\operatorname{ch}^{-1} z\right)}\right\}\left\{-\frac{\operatorname{sh} 2 \sigma \operatorname{ch}\left(k \operatorname{ch}^{-1} t\right)}{\operatorname{sh} 2 k \sigma}\right. \\
& +t \frac{\operatorname{sh}\left(k \operatorname{ch}^{-1} \bar{t}\right)}{\operatorname{sh}\left(\operatorname{ch}^{-1} \bar{t}\right)}-\frac{\operatorname{sh}(2 k-2) \sigma}{\operatorname{sh} 2 k \sigma} \operatorname{ch}\left(k \operatorname{ch}^{-1} \bar{t}\right) \\
& \left.-\operatorname{ch} 2 \sigma \frac{\operatorname{sh}\left((k-1) \operatorname{ch}^{-1} \bar{t}\right)}{\operatorname{sh}\left(\operatorname{ch}^{-1} \bar{t}\right)}\right\} \text {. }
\end{aligned}
$$

We remark that differentiation of (4l) with respect to $t$ yields an explicit formula for

$$
2 \frac{\partial}{\partial z} \Delta_{t} H(z, t)=\frac{\partial \Delta_{t} H}{\partial x}-i \frac{\partial \Delta_{t} H}{\partial y} .
$$


Taking real and imaginary parts and integrating, respectively, with regard to $x$ and $y$, we can derive a formula for $\Delta_{t} H(z, t)$. Once in possession of this quantity, we can solve the boundary value problem for the elastic plate explicitly by means of the Green's formula. For $z=x$ real, we obtain

$$
\begin{aligned}
& \Delta_{t} H(x, t)=4 \log \left|\frac{t-x}{t-\operatorname{ch} \sigma}\right| \\
& +\frac{4}{R \operatorname{sh} 2 \sigma}\left(\operatorname{sh} 2 \sigma \operatorname{ch} \sigma-2 x^{2} \operatorname{sh} \sigma\right)+8 \sum_{k=2}^{\infty} \frac{k}{\operatorname{sh} 2 k \sigma+k \operatorname{sh} 2 \sigma} \\
& \left\{\left[\frac{k^{-2 k}}{2 k(k+1)}+\frac{R^{-2}}{2(k+1)}-\frac{1}{2 k}\right] \operatorname{ch}\left((k+1) \operatorname{ch}^{-1} x\right)\right. \\
& -\left[\frac{k^{-2 k}}{2 k(k-1)}-\frac{R^{2}}{2(k-1)}+\frac{1}{2 k}\right] \operatorname{ch}\left((k-1) \operatorname{ch}^{-1} x\right) \\
& \left.-\frac{R^{-\mathrm{k}}}{2 k}\left[\frac{R}{k-1}-\frac{k^{-1}}{k+1}\right] \cdot(\operatorname{sh} 2 k \sigma+k \operatorname{sh} 2 \sigma)\right\} \\
& \Re\left\{\frac{\operatorname{sh}\left(k \operatorname{ch}^{-1} t\right)}{\operatorname{sh}\left(\operatorname{ch}^{-1} t\right)}\right\}, \quad R=e^{\sigma} \text {. }
\end{aligned}
$$

9. An inequality. We remark at this point that the application of Green's theorem in $\$ 8$ leading to the orthogonality of the functions

$$
\frac{1}{\pi} \frac{\partial U_{k}}{\partial w}=\bar{w} \psi_{k}^{\prime}(w)+F_{k}^{\prime}(w)
$$

was not rigorous, since we did not prove that these functions are square integrable over $D$. We justify the formal manipulations here, thus proving (40) in all detail, and we derive further an extremal property of the eigenfunctions $\bar{w} \psi_{k}{ }^{\prime}+F_{k}{ }^{\prime}$.

We assume, for the sake of simplicity, that $D$ is simply connected, and we assume that $C$ is an analytic curve. We show first that to each square integrable function $\psi(z) \in \Omega$ there corresponds an analytic function $F(z)$ in $D$ with

$$
\iint_{D}\left|\bar{z} \psi^{\prime}(z)+F^{\prime}(z)\right|^{2} d \sigma \leq A \iint_{D}|\psi(z)|^{2} d \sigma
$$


where $A$ is a constant independent of $\psi$. This result is remarkable chiefly because the function $\psi^{\prime}(z)$ itself need not be square integrable.

Let $z(t)$ be a conformal mapping of the unit circle $E$ in the $t$-plane upon $D$. Then by the analyticity of $C, z(t)$ is analytic in the closed unit circle and

$$
z(t)=\sum_{n=0}^{\infty} a_{n t} t^{m}, \quad a_{m}=O\left(\rho^{m}\right), \quad \rho<1 .
$$

In order to prove (44), it is evidently sufficient to show that there is a constant $B$ such that

$$
\iint_{E}\left|\overline{z(t)} \frac{d \psi}{d t}+\frac{d F}{d t}\right|^{2} d \sigma_{t} \leq B \iint_{E}|\psi|^{2} d \sigma_{t} .
$$

By direct computation we obtain

$$
\begin{aligned}
\iint_{E}\left|\bar{t}^{m} t^{k}-\frac{k-m+1}{k+1} t^{k-m}\right|^{2} d \sigma & =\frac{\pi m^{2}}{(m+k+1)(k+1)^{2}}, & k \geq m, \\
\iint_{E}\left|\bar{t}^{m} t^{k}\right|^{2} d \sigma & =\frac{\pi}{m+k+1}, & k<m .
\end{aligned}
$$

If

$$
\psi(t)=\sum_{k=0}^{\infty} \frac{b_{k-1} t^{k}}{k}, \quad|t|<1,
$$

so that

$$
\iint_{E}|\psi|^{2} d \sigma=\pi \sum_{k=0}^{\infty} \frac{\left|b_{k-1}\right|^{2}}{k^{2}(k+1)}<\infty
$$

then we obtain

$$
\begin{aligned}
& \iint_{E}\left|\bar{t}^{m} \frac{d \psi}{d t}-\sum_{k=m}^{\infty} b_{k} \frac{k-m+1}{k+1} t^{k-m}\right|^{2} d \sigma \\
& =\iint_{E}\left|\bar{t}^{m} \sum_{k=0}^{\infty} b_{k} t^{k}-\sum_{k=m}^{\infty} b_{k} \frac{k-m+1}{k+1} t^{k-m}\right|^{2} d \sigma \\
& =\pi \sum_{k=0}^{m-1} \frac{\left|b_{k}\right|^{2}}{m+k+1}+\pi \sum_{k=m}^{\infty}\left|b_{k}\right|^{2} \frac{m^{2}}{(m+k+1)(k+1)^{2}} \leq m^{2} \iint_{E}|\psi|^{2} d \sigma .
\end{aligned}
$$


Hence we make the definition

$$
\frac{d F}{d t}=-\sum_{m=1}^{\infty}-\overline{a_{m}} \sum_{k=m}^{\infty} b_{k} \frac{k-m+1}{k+1} t^{k-m},
$$

so that $d F / d t$ is clearly analytic in $E$, and we have

$$
\begin{aligned}
& \iint_{E}\left|\overline{z(t)} \frac{d \psi}{d t}+\frac{d F}{d t}\right|^{2} d \sigma \\
& \quad=\iint_{E}\left|\sum_{m=0}^{\infty} \sum_{k=0}^{\infty} \bar{a}_{m i} \bar{t}^{m} b_{k} t^{k}-\sum_{m=0}^{\infty} \sum_{k=m}^{\infty} \overline{a_{m}} b_{k} \frac{k-m+1}{k+1} t^{k-m}\right|^{2} d \sigma \\
& \leq \iint_{E}\left\{\sum_{m=0}^{\infty}\left|a_{m}\right| \sum_{m=0}^{\infty}\left|a_{m i}\right|\left|\bar{t}^{m} \sum_{k=0}^{\infty} b_{k} t^{k}-\sum_{k=m}^{\infty} \dot{b}_{k} \frac{k-m+1}{k+1} t^{k-m}\right|^{2}\right\} d \sigma \\
& \leq \sum_{m=0}^{\infty}\left|a_{m}\right| \sum_{m=0}^{\infty} m^{2}\left|a_{m}\right| \iint_{E}|\psi|^{2} d \sigma .
\end{aligned}
$$

Thus (44) is proved.

We now choose $F_{k}$ so that

$$
\iint_{D}\left|\bar{z} \psi_{k}^{\prime}(z)+F_{k}^{\prime}(z)\right|^{2} d \sigma=\text { minimum },
$$

and we obtain

$$
\iint_{D}\left\{\bar{z} \psi_{k}^{\prime}+F_{k}^{\prime}\right\} \bar{\phi} d \sigma=0,
$$

Hence, by the usual argument, the function

$$
V_{k}(w)=-\iint_{D}\left\{\bar{z} \psi_{k}^{\prime}(z)+F_{k}{ }^{\prime}(z)\right\} \frac{d \sigma}{\bar{z}-\bar{w}},
$$

has continuous boundary values 0 on $C$. But

$$
\frac{\partial^{2} V_{k}}{\partial w \partial \bar{w}}=\pi \psi_{k}^{\prime}(w)=\frac{\partial^{2} U_{k}}{\partial w \partial \bar{w}},
$$


and therefore $V_{k} \equiv U_{k}$, since $U_{k}-V_{k}$ is harmonic in $D$ and zero on $C$.

If $p_{m}(z)$ is a polynomial with

$$
\iint_{D}\left|\psi_{k}(z)-p_{m}(z)\right|^{2} d \sigma<\epsilon,
$$

then by (44) there is an analytic function $P_{m}{ }^{\prime}(z)$ such that

$$
\iint_{D}\left|\bar{z} \psi_{k}^{\prime}(z)+F_{k}^{\prime}(z)-\bar{z} p_{m}^{\prime}(z)-P_{m}^{\prime}(z)\right|^{2} d \sigma<A \epsilon .
$$

By Green's theorem, we have, rigorously,

$$
\iint_{D} \frac{\partial U_{l}}{\partial z}\left\{z \overline{p_{m}^{\prime}(z)}+\overline{P_{m}^{\prime}(z)}\right\} d \sigma=-\iint_{D} U_{l}(z) \overline{p_{m}^{\prime}(z)} d \sigma=\iint_{D} \frac{\partial U_{l}}{\partial \bar{z}} \overline{p_{m}(z)} d \sigma,
$$

and, as $\epsilon \longrightarrow 0$, we obtain the desired relation

$$
\iint_{D} \frac{\partial U_{l}}{\partial z} \frac{\partial \bar{U}_{k}}{\partial \bar{z}} d \sigma=\iint_{D} \frac{\partial U_{l}}{\partial \bar{z}} \frac{\partial \bar{U}_{k}}{\partial z} d \sigma=\pi\left\{1-\mu_{k}^{2}\right\} \delta_{k l} .
$$

It is now evident that the functions $\psi_{2}(z)$ and $F_{2}(z)$ solve the extremal problem

$$
\lambda=\frac{\min _{F} \iint_{D}\left|\bar{z} \psi^{\prime}(z)+F^{\prime}(z)\right|^{2} d \sigma}{\iint_{D}|\psi(z)|^{2} d \sigma}=\text { minimum },
$$

among all pairs $\psi \in \Omega$ and $F$ with

$$
\iint_{D} \psi d \sigma=\iint_{D} \bar{\psi}_{1} \psi d \sigma=0 .
$$

For if

$$
\psi=\sum_{k=2}^{\infty} a_{k} \psi_{k}
$$

then

$$
\lambda=\frac{\sum_{k=2}^{\infty}\left|a_{k}\right|^{2}\left(1-\mu_{k}^{2}\right)}{\sum_{k=2}^{\infty}\left|a_{k}\right|^{2}} .
$$


Thus we see that the $\psi_{k}$ are the eigenfunctions and the

$$
\lambda_{k}=1-\mu_{k}^{2}
$$

are the eigenvalues for the eigenvalue problem (45). Therefore (45) is dual to Friedrichs' original eigenvalue problem, and we have, corresponding to his basic inequality

$$
\left|\iint_{D} \psi^{2} d \sigma\right| \leq \mu_{2} \iint_{D}|\psi|^{2} d \sigma, \quad \iint_{D} \psi d \sigma=0, \mu_{2}<1,
$$

the inequality

$$
\lambda_{2} \iint_{D}|\psi|^{2} d \sigma \leq \iint_{D}\left|\bar{z} \psi^{\prime}+F^{\prime}\right|^{2} d \sigma, \quad \iint_{D} \psi d \sigma=0, \lambda_{2}>0,
$$
with the sharp estimates $\mu_{2}$ and $\lambda_{2}$ connected by the relation

$$
\lambda_{2}=1-\mu_{2}^{2} .
$$

While for

$$
\iint_{D}|\psi|^{2} d \sigma=1, \iint_{D} \psi d \sigma=0
$$

we should expect

$$
\iint_{D}\left|\bar{z} \psi^{\prime}+F^{\prime}\right|^{2} d \sigma
$$

to be positive, the inequality (46) states that there is a positive uniform lower bound $\lambda_{2}$ for this integral which depends only upon the geometry of the domain $D$. In summary, we have shown all the following eigenvalue problems to be related.

(a)

$$
\mu=\frac{\left|\iint_{D} \psi^{2} d \sigma\right|}{\iint_{D}|\psi|^{2} d \sigma}=\max ., \text { with } \partial \psi / \partial \bar{z}=0 \text { in } D
$$

(b)

$$
\frac{1+\mu}{1-\mu}=\frac{\iint_{D} u^{2} d \sigma}{\iint_{D} v^{2} d \sigma}=\max ., \text { with } u_{x}=v_{y}, v_{x}=-u_{y} \text { in } D
$$

(c)

$$
1-\mu^{2}=\frac{\iint_{D}\left|\frac{\partial m}{\partial z}\right|^{2} d \sigma}{\iint_{D}\left|\frac{\partial m}{\partial \bar{z}}\right|^{2} d \sigma}=\min ., \text { with } \partial^{2} \mathrm{~m} / \partial \bar{z}^{2}=0 \text { in } D \text {; }
$$


(d)

$$
\mu=\frac{\left|\iint_{D}\left(\frac{\partial U}{\partial \bar{z}}\right)^{2} d \sigma\right|}{\iint_{D}\left|\frac{\partial U}{\partial \bar{z}}\right|^{2} d \sigma}=\max \text {, with } U \equiv 0 \text { on } C
$$

$$
\mu \frac{\partial^{2} \bar{U}}{\partial z \partial \bar{z}}+\frac{\partial^{2} U}{\partial \bar{z}^{2}}=0 \text {, with } U \equiv 0 \text { on } C \text {. }
$$

The eigenfunctions $\psi_{k}$ of (a) are obviously [7] connected with the eigenfunctions $u_{k}, v_{k}$ of (b) by the relation $\psi_{k}=u_{k}+i v_{k}$. Problem (c) is merely a restatement of (45), and (d) is the Euler problem immediately associated with (e) by the Dirichlet principle. We have, in fact

$$
\begin{aligned}
& \iint_{D}\left(\frac{\partial U_{k}}{\partial \bar{z}}\right)^{2} d \sigma=\pi^{2} \iint_{D}\left(\mu_{k} \bar{\psi}_{k}-\psi_{k}\right)^{2} d \sigma=\pi^{2} \mu_{k}\left(\mu_{k}^{2}-1\right), \\
& \iint_{D}\left|\frac{\partial U_{k}}{\partial \bar{z}}\right|^{2} d \sigma=\pi^{2} \iint_{D}\left|\mu_{k} \bar{\psi}_{k}-\psi_{k}\right|^{2} d \sigma=\pi^{2}\left(1-\mu_{k}^{2}\right) .
\end{aligned}
$$

Thus one of our leading results is the relation between the extremal problems (a) and (c) with differential side conditions and the extremal problem (d) with a boundary condition. The free boundary problems (a) and (c) naturally replace the classical problem (d) in our present discussion of the existence of solutions of (e).

\section{REFERENCES}

1. S. Bergman, Sur les fonctions orthogonales de plusieurs variables complexes, avec les applications à la théorie des fonctions analytiques, Gauthier-Villars, Paris, 1947.

2. Z Zwei Sätze über Funktionen von zwei komplexen Veränderlichen, Math. Ann. 100 (1928), 399-410.

3. S. Bergman and M. Schiffer, A representation of Green's and Neumann's functions in the theory of partial differential equations of second order, Duke Math. J. 14 (1947), 609-638.

4. - On Green's and Neumann's functions in the theory of partial differential equations, Bull. Amer. Math. Soc. 53 (1947), 1141-1151.

5. - Kernel functions in the theory of partial differential equations of elliptic type, Duke Math. J. 15 (1948), 535-566.

6. , Kernel functions and conformal mapping, to appear in the Duke Math. J.

7. K. O. Friedrichs, On certain inequalities and characteristic value problems for analytic functions and for functions of two variables, Trans. Amer. Math. Soc. 41 (1937), 321-364. 
8. P. R. Garabedian, Schwarz's lemma and the Szegö kernel function, Trans. Amer. Math. Soc. 67 (1949), 1-35.

9. , A new proof of the Riemann mapping theorem, to appear shortly.

10. P. R. Garabedian and M. Schiffer, On existence theorems of potential theory and conformal mapping, Ann. of Math. 52 (1950), 164-187.

11. J. Hadamard, Mémoire sur le problème d'analyse relatif à l'équilibre des plaques élastiques encastrées, Mémoires présentés par divers savants à l'Académie des Sciences, (2) 33 (1908).

12. O. Lehto, Anwendung orthogonaler Systeme auf gewisse funktionentheoretische Extremal-und Abbildungsprobleme, Ann. Acad. Sci. Fennicae, Ser. A. I. Math.-Phys. no. 59 (1949).

13. M. Schiffer, The kernel function of an orthonormal system, Duke Math. J. 13 (1946), 529-540.

14. M. Schiffer and G. Szegö, Virtual mass and polarization, Trans. Amer. Math. Soc. 67 (1949), 130-205.

15. G. Szegö, Orthogonal polynomials, Amer. Math. Soc. Coll. Pub., vol. 23, New York, 1939.

16. J. L. Walsh, Interpolation and approximation by rational functions in the complex domain, Amer. Math. Soc. Coll. Pub., vol. 20, New York, 1935.

17. S. Zaremba, L'équation biharmonique et une class remarquable de fonctions fondamentales harmoniques, Bull. International de l'Académie des Sciences de Cracovie (1907), 147-196.

18. - Sur le calcul numérique des fonctions demandées dans le problème de Dirichlet et le probleme hydrodynamique, Bull. International de l'Academie des Sciences de Cracovie (1908), 125-195.

19. G. Fichera, Sull'esistenza delle funzioni potenziali nei problemi della fisica matematica, Atti Accad. Naz. Lincei. Rend. Cl. Sci. Fis. Mat. Nat. (8) 2, (1947), 527-532.

20. , Teorema d'esistenza per il problema bi-iperarmonico, Atti Accad. Naz. Lincei. Rend. Cl. Sci. Fis. Mat. Nat. (8) 5 (1948), 319-324.

21. , On some general integration methods employed in connection with linear differential equations, J. of Math. and Phys., vol. 31 (1950), 59-68.

\section{STANFORD UNIVERSITY}




\section{PACIFIC JOURNAL OF MATHEMATICS}

EDITORS

\author{
M.M. SCHIFFER* \\ Stanford University \\ Stanford, California \\ E. HEWITT \\ University of Washington \\ Seattle 5, Washington
}

\author{
R.P. DILWORTH \\ California Institute of Technology \\ Pasadena 4, California \\ E.F. BECKENBACH** \\ University of California \\ Los Angeles 24, California
}

\section{ASSOCIATE EDITORS}

$\begin{array}{llll}\text { H. BUSEMANN } & \text { P.R. HALMOS } & \text { BØRGE JESSEN } & \text { J. J. STOKER } \\ \text { HERBERT FEDERER } & \text { HEINZ HOPF } & \text { PAUL LÉVY } & \text { E.G. STRAUS } \\ \text { MARSHALL HALL } & \text { R.D. JAMES } & \text { GEORGE PÓLYA } & \text { KÔSAKU YOSIDA }\end{array}$

\section{SPONSORS}

UNIVERSITY OF BRITISH COLUMBIA

UNIVERSITY OF SOUTHERN CALIFORNIA

CALIFORNIA INSTITUTE OF TECHNOLOGY

UNIVERSITY OF CALIFORNIA, BERKELEY

STANFORD RESEARCH INSTITUTE

UNIVERSITY OF CALIFORNIA, DAVIS

STANFORD UNIVERSITY

UNIVERSITY OF CALIFORNIA, LOS ANGELES

UNIVERSITY OF CALIFORNIA, SANTA BARBARA

WASHINGTON STATE COLLEGE

UNIVERSITY OF WASHINGTON

UNIVERSITY OF NEVADA

OREGON STATE COLLEGE

AMERICAN MATHEMATICAL SOCIETY

UNIVERSITY OF OREGON

HUGHES AIRCRAFT COMPANY

Mathematical papers intended for publication in the Pacific Journal of Mathematics should be typewritten (double spaced), and the author should keep a complete copy. Manuscripts may be sent to any. of the editors. Manuscripts intended for the outgoing editors should be sent to their successors. All other communications to the editors should be addressed to the managing editor, E.G. Straus, at the University of California Los Angeles 24, California.

50 reprints of each article are furnished free of charge; additional copies may be obtained at cost in multiples of 50 .

The Pacific Journal of Mathematics is published quarterly, in March, June, September, and December. The price per volume (4 numbers) is $\$ 12.00$; single issues, $\$ 3.50$; back numbers (Volumes $1,2,3$ ) are available at $\$ 2.50$ per copy. Special price to individual faculty members of supporting institutions and to individual members of the American Mathematical Society: $\$ 4.00$ per volume; single issues, $\$ 1.25$.

Subscriptions, orders for back numbers, and changes of address should be sent to the publishers, University of California Press, Berkeley 4, California.

Printed at Ann Arbor, Michigan. Entered as second class matter at the Post Office, Berkeley, California.

* To be succeeded in 1955, by H.L. Royden, Stanford University, Stanford, California.

** To be succeeded in 1955, by E.G. Straus, University of California, Los Angeles 24, Calif.

UNIVERSITY OF CALIFORNIA PRESS - BERKELEY AND LOS ANGELES

COPYRIGHT 1954 BY PACIFIC JOURNAL OF MATHEMATICS 


\section{Pacific Journal of Mathematics}

\section{Vol. 1, No. $4 \quad, 1951$}

R. H. Bruck, Loops with transitive automorphism groups ............ 481

Paul R. Garabedian, A partial differential equation arising in conformal

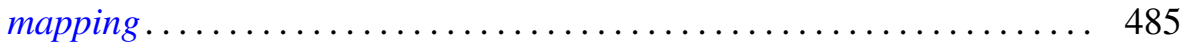

Magnus R. Hestenes, Applications of the theory of quadratic forms in Hilbert space to the calculus of variations .................. 525

Sze-Tsen Hu, On the realizability of homotopy groups and their operations ..................................... 583 